\title{
Weakly nonlinear dynamics in noncanonical Hamiltonian systems with applications to fluids and plasmas
}

\author{
P. J. Morrison ${ }^{\dagger}$ and J. Vanneste ${ }^{\ddagger}$ \\ $\dagger$ Dept. of Physics and Institute for Fusion Studies, University of Texas, Austin, USA \\ $¥$ School of Mathematics and Maxwell Institute for Mathematical Sciences, University of \\ Edinburgh, UK
}

\begin{abstract}
A method, called beatification, is presented for rapidly extracting weakly nonlinear Hamiltonian systems that describe the dynamics near equilibria for systems possessing Hamiltonian form in terms of noncanonical Poisson brackets. The procedure applies to systems like fluids and plasmas in terms of Eulerian variables that have such noncanonical Poisson brackets, i.e., brackets with nonstandard and possibly degenerate form. A collection of examples of both finite and infinite dimensions is presented.
\end{abstract}

\section{Introduction}

The most important physical systems are either governed by evolution equations that are, in one sense or another, Hamiltonian or possess Hamiltonian limits in which dissipative or transport terms associated with phenomenological constants are dropped. This imposes strong constraints on their dynamics with, for instance the existence of differential invariants, and the possibility of symmetryrelated conservation laws. The existence of a Hamiltonian structure is particularly important for perturbative problems. The structure can not only be exploited to simplify asymptotic developments considerably; it also leads to several results, such as the adiabatic invariance of action, without counterparts in non-Hamiltonian systems.

Hamiltonian perturbation has been largely developed for canonical systems, with equations

$$
\dot{p}_{a}=\frac{\partial H}{\partial q^{a}}=\left\{p_{a}, H\right\}, \quad \dot{q}^{a}=-\frac{\partial H}{\partial p_{a}}=\left\{q^{a}, H\right\},
$$

associated with the Hamiltonian $H$ and canonical Poisson bracket

$$
\{f, g\}=\frac{\partial f}{\partial p_{a}} \frac{\partial g}{\partial q^{a}}-\frac{\partial g}{\partial p_{a}} \frac{\partial f}{\partial q^{a}},
$$

where $a=1 \ldots, N$ and repeated indices here and henceforth are summed. The perturbative methods typically rely on canonical transformation, which, after truncation, simplify the Hamiltonian while leaving the form of the Poisson bracket unchanged. This is particularly efficient since the computation is focussed on a scalar - the Hamiltonian — rather than on the evolution equations themselves. The approach is well illustrated by the classical results on averaging, passage through resonance, adiabatic invariance, etc. (e.g. [1, 2]).

Here, we are interested in the more general class of noncanonical Hamiltonian systems (a terminology introduced [3], see also e.g. $[4,5])$ that have the form

$$
\dot{z}^{i}=J^{i j}(z) \frac{\partial H}{\partial z^{j}}=\left\{z^{i}, H\right\},
$$

where $H$ is the Hamiltonian, $i, j=1,2, \ldots, M$, and $J^{i j}$ denotes the components of the Poisson matrix, which is skew-symmetric, satisfies the Jacobi identity

$$
J^{i k} \frac{\partial J^{j l}}{\partial z^{k}}+J^{j k} \frac{\partial J^{l i}}{\partial z^{k}}+J^{l k} \frac{\partial J^{i j}}{\partial z^{k}}=0,
$$

1

(C) 2016. This manuscript version is made available under the Elsevier user license

http://www.elsevier.com/open-access/userlicense/1.0/ 
and defines the Poisson bracket

$$
\{f, g\}=\frac{\partial f}{\partial z^{i}} J^{i j} \frac{\partial g}{\partial z^{j}} .
$$

In general, $J^{i j}$ depends on $z$ and is often degenerate (singular), unlike the canonical form where it is given by

$$
J_{c}=\left(\begin{array}{rr}
0_{N} & I_{N} \\
-I_{N} & 0_{N}
\end{array}\right),
$$

with $I_{N}$ the $N \times N$ identity matrix and $0_{N}$ an $N \times N$ block of zeros. Substitution of (1.5) into (1.4) yields (1.1).

For the degenerate case, it was known by S. Lie (see e.g. $[6,7]$ ) that the null-space of $J$ is spanned by the gradients of the Casimir functionals $C^{\alpha}$ (referred to as distinguished functions by Lie) that satisfy

$$
J^{i j} \frac{\partial C^{\alpha}}{\partial z^{j}}=0, \quad \alpha=1,2, \ldots, M-2 N,
$$

where $2 N$ is the rank of $J^{i j}$. Note, we will systematically use greek symbols to index the Casimirs functionals in what follows. Systems of the form (1.2) emerge in many areas of physics, such as fluid and plasma models in terms of Eulerian variables. They are typically obtained from canonical systems after symmetry reduction, and have therefore the advantage of a state space of reduced dimensionality $[4,5,8,9]$.

However, when in comes to the development of perturbation theories, the form (1.2) is generally considered inconvenient, notwithstanding the efficacious use of noncanonical coordinates for perturbation theory in some cases [10]. The difficulty stems from the $z$-dependence of $J^{i j}(z)$ : when $z$ is expanded, for instance in power series of a small parameter, $J^{i j}(z)$ also needs to be expanded. If this expansion is then truncated, the Jacobi identity (1.3) then usually ceases to hold, and the system loses its Hamiltonian nature.

In this paper, we discuss how this difficulty can be overcome in order to study perturbatively the weakly nonlinear dynamics of noncanonical systems in the neighbourhood of a (stable) equilibrium $z_{0}$. The idea is to perform a near identity change of variables that transforms $J^{i j}(z)$ into its (constant) value $J^{i j}\left(z_{0}\right)$ at the equilibrium. Because transforming the Poisson matrix to a constant, i.e., a form independent of $z$, is the first step of canonization, transforming to canonical form, we refer to this procedure as beatification. This change of variables is then introduced in the Hamiltonian, which can be truncated. The result is a Hamiltonian system with constant (but noncanonical) Poisson matrix, where nonlinearity has been transferred from the Poisson bracket to the Hamiltonian. This system can then be studied perturbatively, by applying further changes of variables that either leave the constant Poisson matrix invariant or transform it into the canonical form of (1.5), which is known to be always possible by Darboux's theorem (e.g. [11]). Here, we provide a practical implementation for systems near an equilibrium. In principle, the approach can be carried out order by order in the small parameter that measures the size of the perturbation away from the equilibrium. We limit our discussion to the first step in the procedure. This is sufficient to describe the leading-order effect of the nonlinearity and study effects such as quadratic resonance and possible explosive instabilities, a special case of which was considered in detail in [12].

We note, the linearization problem of literature $[13,14,15]$ of smoothly transforming general brackets to local Lie-Poisson form, differs from the procedure here: our goal is to 'flatten' brackets to first order and thereby remove the linear dependence replacing it by a constant.

The paper is organized as follows. In Section 2 we consider finite-dimensional systems of the form (1.2). For these, an explicit formula is given for the change of variable that makes the Poisson matrix constant to leading order. We consider both the cases of nondegenerate and degenerate Poisson matrices, and we give a simplified formulation for systems with Lie-Poisson structure. Applications based on the real semi-simple Lie algebras $\mathfrak{s o}(2,1)$ and $\mathfrak{s o}(3)$, where the former applies to the Kida vortex and the latter to the rigid body, the heavy top, and a two-spin system, are presented in Section 3 to illustrate the method. Infinite-dimensional systems are discussed in Section 4. For these, we do not give a general form for the change of variables, which would be overly complicated. Rather, we derive the transformation explicitly for Poisson brackets of fluid dynamics and plasma physics when the equilibrium depends on only a single coordinate. In Section 5 we first consider the Lie-Poisson bracket for the two-dimensional Euler equation that describes the ideal fluid, which is identical to the bracket that describes the one-dimensional Vlasov-Poisson and other systems. Then, 
in Section 6, we consider the bracket for two-dimensional stratified fluids and for two-dimensional magnetohydrodynamics. For these four physical systems, we implement the relevant change of variables in the Hamiltonian and derive the equations of motion in the transformed variables. These equations are suited for the study of the weakly nonlinear interactions of waves or more generally modes, for instance in the statistical treatment of weak turbulence (e.g. [16, 17]). We conclude in Section 7 where we summarize and discuss our results and consider some future applications. The paper also contains several appendices that expand upon material presented in the main text. In particular, Appendix E demonstrates how a canonical structure for the weakly nonlinear VlasovPoisson equation can be obtained by building on the coordinate transformation of Section 6 and on earlier work on the canonization of the linearized system.

\section{Finite-dimensional systems}

Our treatment of finite-dimensional systems is divided into three subsections. In Section 2.1 we set up the basic expansion formulation, in preparation for Section 2.2 where beatification is explicitly undertaken. Then, in Section 2.3 we consider the special case of Lie-Poisson systems where the initial Poisson vector is linear in $z$.

\subsection{Formulation}

An equilibrium $z_{0}$ of (1.2) satisfies

$$
\dot{z}^{i}=J_{0}^{i j} \frac{\partial H_{0}}{\partial z_{0}^{j}}:=J^{i j}\left(z_{0}\right) \frac{\partial H\left(z_{0}\right)}{\partial z_{0}^{j}}=0 .
$$

Provided that the rank of $J^{i j}$ does not change at $z_{0}, z_{0}$ is a critical point of the combination

$$
F(z):=H(z)+\lambda_{\alpha}\left(z_{0}\right) C^{\alpha}(z)
$$

for some $\lambda_{\alpha}$, i.e.

$$
\frac{\partial F\left(z_{0}\right)}{\partial z_{0}^{j}}=0
$$

To study the evolution of a small disturbance to $z_{0}$, we write

$$
z=z_{0}+\epsilon z^{\prime}
$$

where $\epsilon \ll 1$. In what follows, we will omit the prime, using $z$ for the disturbance. Its evolution equation is governed by

$$
\dot{z}^{i}=J_{z}^{i j} \frac{\partial F_{z}}{\partial z^{j}}
$$

where

$$
J_{z}^{i j}:=J^{i j}\left(z_{0}+\epsilon z\right) \quad \text { and } \quad F_{z}:=\epsilon^{-2}\left[F\left(z_{0}+\epsilon z\right)-F\left(z_{0}\right)\right] .
$$

The Hamiltonian $F_{z}$, which we will refer to as the free energy is sometimes called the pseudoenergy. The $O(\epsilon)$ approximation of this equation reads

$$
\dot{z}^{i}=\left(J_{0}^{i j}+\epsilon \frac{\partial J_{0}^{i j}}{\partial z_{0}^{l}} z^{l}\right)\left(\frac{\partial^{2} F_{0}}{\partial z_{0}^{j} \partial z_{0}^{k}} z^{k}+\frac{\epsilon}{2} \frac{\partial^{3} F_{0}}{\partial z_{0}^{j} \partial z_{0}^{k} \partial z_{0}^{m}} z^{k} z^{m}\right)+O\left(\epsilon^{2}\right) .
$$

In general, this equation is not Hamiltonian: due to the truncation of $J_{z}^{i j}$ as

$$
J_{0}^{i j}+\epsilon \frac{\partial J_{0}^{i j}}{\partial z_{0}^{l}} z^{l},
$$

the Jacobi identity is not satisfied. However, through a change of variable, it is possible to find a new equation, equivalent to (2.4) up to $O\left(\epsilon^{2}\right)$, that is Hamiltonian. Let $\eta=\eta(z)$ be the new dependent variable. The corresponding Poisson matrix is given by

$$
J_{\eta}^{i j}=\frac{\partial \eta^{i}}{\partial z^{k}} J_{z}^{k l} \frac{\partial \eta^{j}}{\partial z^{l}}
$$


We will choose $\eta$ such that

$$
J_{\eta}^{i j}=J_{0}^{i j}+O\left(\epsilon^{2}\right)
$$

In contrast to the truncation of $J_{z}^{i j}$ given by (2.5), the truncation of $J_{\eta}^{i j}$ to $O(\epsilon)$ satisfies the Jacobi identity: indeed, it is simply given by $J_{0}^{i j}$ and thus has constant coefficients, making satisfaction of (1.3) immediate. The evolution equation for $\eta$ takes the Hamiltonian form

$$
\dot{\eta}^{i}=J_{0}^{i j} \frac{\partial F_{\eta}}{\partial \eta^{j}}+O\left(\epsilon^{2}\right)
$$

where the Hamiltonian

$$
F_{\eta}:=\epsilon^{-2}\left[F\left(z_{0}+\epsilon z(\eta)\right)-F\left(z_{0}\right)\right]
$$

can be consistently truncated to $O(\epsilon)$.

\subsection{Beatification: flattening the Poisson matrix}

We now construct the new variable $\eta$ leading to (2.7). The change of variable is clearly near-identity so that, to the order of interest, it can be written

$$
\eta^{i}=z^{i}+\frac{\epsilon}{2} D_{k l}^{i} z^{k} z^{l}+O\left(\epsilon^{2}\right)
$$

where the quantity $D^{i}{ }_{k l}$ is symmetric in $k l$. Introducing this expression in (2.6), we can rewrite the condition (2.7) as

$$
S_{l}^{i j}{ }_{l}:=J_{0}^{i k} D_{k l}^{j}+J_{0}^{k j} D^{i}{ }_{k l}+\frac{\partial J_{0}^{i j}}{\partial z_{0}^{l}}=0 .
$$

Because of the skew-symmetry of $J_{0}^{i j}$, this system contains a maximum of $M^{2}(M-1) / 2$ independent equations, while $D^{i}{ }_{k l}$ contains $M^{2}(M+1) / 2$ coefficients, and their difference, $M^{2}$, arises because of the nonuniqueness of the variables we seek. Equation (2.10) is akin to the formula obtained in Riemannian geometry when one seeks coordinates, so-called normal coordinates, in which the metric is flat (e.g. [18]). In this case the quantities analogous to $D^{i}{ }_{k l}$ turn out to be the Christoffel symbols. In our case, we will see that the situation is somewhat more complicated.

To solve (2.10) for $D_{k l}^{i}$, we introduce the following decomposition:

$$
D_{k l}^{i}=\frac{1}{3} \frac{\partial J_{0}^{i m}}{\partial z_{0}^{k}} \omega_{l m}+\frac{1}{3} \frac{\partial J_{0}^{i m}}{\partial z_{0}^{l}} \omega_{k m}+\hat{D}_{k l}^{i}
$$

where $\omega_{l m}$ and $\hat{D}^{i}{ }_{k l}$ are yet to be determined; $\omega_{l m}$ is assumed to be skew-symmetric, while $\hat{D}^{i}{ }_{k l}$ is symmetric in $k l$. Introducing expression (2.11) into (2.10) yields

$$
\begin{aligned}
S_{l}^{i j}=\frac{1}{3} \frac{\partial J_{0}^{i j}}{\partial z_{0}^{k}}\left(\delta^{k}{ }_{l}-J_{0}^{k m} \omega_{m l}\right)+ & \frac{1}{3} \frac{\partial J_{0}^{i m}}{\partial z_{0}^{l}}\left(\delta^{j}{ }_{m}-J_{0}^{j k} \omega_{k m}\right)+\frac{1}{3} \frac{\partial J_{0}^{m j}}{\partial z_{0}^{l}}\left(\delta^{i}{ }_{m}-J_{0}^{i k} \omega_{k m}\right) \\
& +J_{0}^{i k} \hat{D}^{j}{ }_{k l}+J_{0}^{k j} \hat{D}^{i}{ }_{k l}=0 .
\end{aligned}
$$

We solve this relation for both degenerate and nondegenerate $J_{0}^{i j}$.

When $J_{0}^{i j}$ is nondegenerate we can immediately enforce $S^{i j}{ }_{l}=0$ by setting

$$
J_{0}^{k l} \omega_{l m}=\delta^{k}{ }_{m},
$$

and $\hat{D}^{i}{ }_{k l}=0$. In this case the quantity $\omega_{l m}$ denotes the components of the usual symplectic two-form dual to the cosymplectic form $J_{0}^{k l}$.

When $J_{0}^{i j}$ is degenerate, however, (2.13) cannot be solved, because $\delta^{k}{ }_{l}$ projects onto the nullspace of $J_{0}^{k m}$. Nevertheless, $\omega_{m l}$ can be chosen as a generalized inverse [19] of $J_{0}^{k m}$, i.e. as solution of the underdetermined system

$$
J_{0}^{k l} \omega_{l m}=\delta^{k}{ }_{m}-\chi_{\alpha}^{k} \chi_{m}^{\alpha} .
$$

Here, the covariant vectors

$$
\chi_{l}^{\alpha}:=\frac{\partial C^{\alpha}\left(z_{0}\right)}{\partial z_{0}^{l}},
$$


span the null space of $J_{0}^{k l}$, while the contravariant vectors $\chi_{\alpha}^{k}$ are defined as their duals, i.e. they satisfy the bi-orthogonality (pairing) relation

$$
\chi_{\beta}^{l} \chi_{l}^{\alpha}=\delta_{\beta}^{\alpha} .
$$

Observe, the right-hand-side of (2.14) no longer contains components in the null space of $J_{0}^{k m}$ and so an inverse is possible.

For $\hat{D}_{k l}^{i}$, we choose

$$
\hat{D}_{k l}^{i}=\frac{1}{3} \frac{\partial \chi_{l}^{\alpha}}{\partial z_{0}^{k}} \chi_{\alpha}^{i}+A_{\alpha k}^{i} \chi_{l}^{\alpha}+A_{\alpha l}^{i} \chi_{k}^{\alpha},
$$

where the coefficients $A_{\alpha k}^{i}$ will be determined later. Note that $\hat{D}_{k l}^{i}$ is symmetric in $k l$ as required, because

$$
\frac{\partial \chi_{l}^{\alpha}}{\partial z_{0}^{k}}=\frac{\partial^{2} C^{\alpha}}{\partial z_{0}^{l} \partial z_{0}^{k}}=\frac{\partial \chi_{k}^{\alpha}}{\partial z_{0}^{l}}
$$

Introducing (2.14) and (2.17) in (2.12), and using (2.18), (2.15) and its consequence

$$
\frac{\partial J_{0}^{i m}}{\partial z_{0}^{l}} \chi_{m}^{\alpha}=-J_{0}^{i m} \frac{\partial \chi_{m}^{\alpha}}{\partial z_{0}^{l}}
$$

yields

$$
\chi_{l}^{\alpha}\left(J_{0}^{i k} A_{\alpha k}^{j}+J_{0}^{k j} A_{\alpha k}^{i}-\frac{1}{3} \frac{\partial J_{0}^{i j}}{\partial z_{0}^{k}} \chi_{\alpha}^{k}\right)=0 .
$$

Using the same properties as previously, it can be checked that for $A_{\alpha k}^{i}$ defined by

$$
A_{\alpha k}^{i}=-\frac{1}{6} \frac{\partial J_{0}^{i n}}{\partial z_{0}^{m}} \omega_{n k} \chi_{\alpha}^{m}+\frac{1}{6} \chi_{\beta}^{i} \frac{\partial \chi_{k}^{\beta}}{\partial z_{0}^{m}} \chi_{\alpha}^{m}
$$

the factor between parenthesis in (2.20) vanishes identically.

We have thus constructed a general solution of (2.10). Collecting (2.11), (2.17), and (2.21), we can write $D^{i}{ }_{k l}$ in the form:

$$
D_{k l}^{i}=\frac{1}{3} \frac{\partial J_{0}^{i n}}{\partial z_{0}^{m}} \omega_{k n}\left(\delta_{l}^{m}+\frac{1}{2} \chi_{\alpha}^{m} \chi_{l}^{\alpha}\right)+\frac{1}{6} \chi_{\beta}^{i} \frac{\partial \chi_{k}^{\beta}}{\partial z_{0}^{m}}\left(\delta_{l}^{m}+\chi_{\alpha}^{m} \chi_{l}^{\alpha}\right)+(k \leftrightarrow l),
$$

where $k \leftrightarrow l$ designates the symmetric term in $k l$.

So far, we have only considered the Poisson matrix, and not the Hamiltonian. The change of variable (2.9) needs to be inverted to compute the Hamiltonian $F_{\eta}$. To the required order of accuracy, the inversion is immediate, and gives

$$
z^{i}=\eta^{i}-\frac{\epsilon}{2} D_{k l}^{i} \eta^{k} \eta^{l}+O\left(\epsilon^{2}\right)
$$

\subsection{Lie-Poisson systems}

Typically dynamical systems that describe matter have Poisson matrices with Lie-Poisson form, which in finite dimensions is given

$$
J^{i j}=c_{k}^{i j} z^{k}
$$

where the $c_{k}^{i j}$ are the structure constants of some Lie algebra. In general, such Lie-Poisson systems can be flattened by the procedure of Section 2.2 .

In the case where the algebra with structure constants $c^{i j}{ }_{k}$ is semisimple [20, 21], the flattening transformation can be found more directly. For semisimple Lie algebras, the Cartan-Killing symmetric tensor,

$$
g^{i j}:=-c_{l}^{i k} c_{k}^{l j}
$$

has an ordinary inverse $g_{i j}$, i.e.

$$
g_{i k} g^{k j}=\delta_{i}{ }^{j}
$$


The 'metric' tensor $g^{i j}$ can then be used to put the structure constants in a fully antisymmetric form $c^{i j k}$ that satisfies

$$
c^{i j k}=c_{l}^{i j} g^{l k} .
$$

It can also be used to relate the covariant and contravariant vectors $\chi_{i}^{\alpha}$ and $\chi_{\alpha}^{i}$ through an expression of the form

$$
\chi_{\alpha}^{i}=f_{\alpha \beta} g^{i k} \chi_{k}^{\beta},
$$

for some symmetric tensor $f_{\alpha \beta}$. Exploiting this expansion, one can find a direct solution of $(2.20)$ given by

$$
A_{\alpha k}^{i}=-\frac{1}{6} f_{\alpha \beta} g^{i m} \frac{\partial \chi_{k}^{\beta}}{\partial z_{0}^{m}}
$$

Indeed, using (2.24)-(2.26), (2.19) and

$$
\frac{\partial J_{0}^{i m}}{\partial z_{0}^{l}}=c_{l}^{i m}
$$

one can find

$$
\begin{aligned}
J_{0}^{i k} A_{\alpha k}^{j}+J_{0}^{k j} A_{\alpha k}^{i} & =\frac{1}{6}\left(c^{i k} g^{j m}+c_{m}^{k j} g^{i m}\right) f_{\alpha \beta} \chi_{k}^{\beta} \\
& =-\frac{1}{3} c^{i j k} f_{\alpha \beta} \chi_{k}^{\beta}=-\frac{1}{3} c^{i j k} g_{l k} \chi_{\alpha}^{l} \\
& =-\frac{1}{3} c^{i j}{ }_{l} \chi_{\alpha}^{l}=-\frac{1}{3} \frac{\partial J_{0}^{i j}}{\partial z_{0}^{l}} \chi_{\alpha}^{l},
\end{aligned}
$$

so that (2.20) is satisfied. Therefore, the change of variable (2.9) is defined by

$$
D_{k l}^{i}=\frac{1}{3} c_{l}^{i m} \omega_{k m}+\frac{1}{6} f_{\alpha \beta} g^{i m}\left(\chi_{m}^{\beta} \frac{\partial \chi_{k}^{\alpha}}{\partial z_{0}^{l}}-\chi_{l}^{\alpha} \frac{\partial \chi_{k}^{\beta}}{\partial z_{0}^{m}}\right)+(k \leftrightarrow l) .
$$

Semisimple Lie-Poisson brackets possess a quadratic Casimir invariant given by

$$
C=g_{i j} z^{i} z^{j}
$$

which we record here for later use.

\section{Applications}

\subsection{Three-dimensional semisimple Lie-Poisson brackets}

It is well-known that there are nine real Lie algebras of dimension three [20]. In this subsection we consider the Lie-Poisson brackets associated with two of these, $\mathfrak{s o}(3)$ and $\mathfrak{s o}(2,1)$, both of which are semisimple. The bracket determined by $\mathfrak{s o}(3)$ describes, e.g., spin systems and Euler's equations for the free rigid body [22], while $\mathfrak{s o}(2,1)$ emerges naturally from quadratic moment projections of Euler's fluid equations and describes, e.g., Kida vortex dynamics [23, 24] .

The rotation algebra $\mathfrak{s o}(3)$ has structure constants $c_{k}^{i j}=\varepsilon_{i j k}$, with the $\varepsilon_{i j k}$ being the purely antisymmetric Levi-Civita tensor, while the structure constants of $\mathfrak{s o}(2,1)$ are the same except for a sign flip. Both Poisson matrices are expressed by the following:

$$
J_{0}^{ \pm}=\left(\begin{array}{ccc}
0 & z_{0}^{3} & -z_{0}^{2} \\
-z_{0}^{3} & 0 & \pm z_{0}^{1} \\
z_{0}^{2} & \mp z_{0}^{1} & 0
\end{array}\right)
$$

where the upper sign corresponds to $\mathfrak{s o}(3)$ and the lower to $\mathfrak{s o}(2,1)$.

For these semisimple algebras we can identify the algebras with there duals by their CartanKilling forms, which we scale as $g^{i j}=\mp 2 \delta_{ \pm}^{i j}$ to give the following metrics:

$$
\delta^{ \pm}=\left(\begin{array}{ccc} 
\pm 1 & 0 & 0 \\
0 & 1 & 0 \\
0 & 0 & 1
\end{array}\right)
$$


where again the upper sign corresponds to $\mathfrak{s o}(3)$ and the lower to $\mathfrak{s o}(2,1)$.

In terms of $\delta^{ \pm}$the structure constants for both cases can be represented as

$$
c_{k}^{i j}=\varepsilon^{i j s} \delta_{s k}^{ \pm} .
$$

Using (3.2) the quadratic Casimirs take the form

$$
\left|z_{0}\right|_{ \pm}^{2}:=\delta_{i j}^{ \pm} z_{0}^{i} z_{0}^{j}=:\left\langle z_{0}, z_{0}\right\rangle_{ \pm}
$$

with the corresponding null eigenvectors and their duals given by

$$
\chi_{i}=\frac{\delta_{i j}^{ \pm} z_{0}^{j}}{\left|z_{0}\right|_{ \pm}} \quad \text { and } \quad \chi^{i}=\frac{z_{0}^{i}}{\left|z_{0}\right|_{ \pm}}
$$

Solving (2.14), one finds that the generalized inverses of the two matrices are given by

$$
\omega^{ \pm}=\frac{1}{\left|z_{0}\right|_{ \pm}^{2}}\left(\begin{array}{ccc}
0 & -z_{0}^{3} & z_{0}^{2} \\
z_{0}^{3} & 0 & -z_{0}^{1} \\
-z_{0}^{2} & z_{0}^{1} & 0
\end{array}\right),
$$

which can easily be checked by substituting (3.4) into (2.14) and making use of (3.3). Observe $\omega_{i j}^{ \pm}=-\epsilon_{i j k} z^{k} /\left|z_{0}\right|_{ \pm}^{2}$.

The different terms of $(2.27)$ can now be evaluated for $\mathfrak{s o}(3)$ and $\mathfrak{s o}(2,1)$ together,

$$
\begin{aligned}
\frac{1}{3} c_{l}^{i m} \omega_{k m} & =\frac{z_{0}^{r}}{3\left|z_{0}\right|_{ \pm}^{2}} \varepsilon^{i m s} \delta_{s l}^{ \pm} \varepsilon_{m k r}=\frac{1}{3\left|z_{0}\right|_{ \pm}^{2}}\left(z_{0}^{i} \delta_{k l}^{ \pm}-z_{0}^{r} \delta_{k}^{i} \delta_{r l}^{ \pm}\right), \\
\frac{1}{6} f_{\alpha \beta} g^{i m}\left(\chi_{m}^{\alpha} \frac{\partial \chi_{k}^{\beta}}{\partial z_{0}^{l}}-\chi_{l}^{\alpha} \frac{\partial \chi_{k}^{\beta}}{\partial z_{0}^{m}}\right) & =\frac{z_{0}^{r}}{6\left|z_{0}\right|_{ \pm}^{2}} \delta_{ \pm}^{i m}\left(\delta_{m r}^{ \pm} \delta_{k l}^{ \pm}-\delta_{l r}^{ \pm} \delta_{k m}^{ \pm}\right)=\frac{1}{6\left|z_{0}\right|_{ \pm}^{2}}\left(z_{0}^{i} \delta_{k l}^{ \pm}-z_{0}^{r} \delta_{i k} \delta_{l r}^{ \pm}\right),
\end{aligned}
$$

where $f_{ \pm}=\mp 1 / 2$ follows from $\chi^{i}=f_{ \pm} g_{ \pm}^{i k} \chi_{k}$. Combining these results leads to

$$
D_{k l}^{i}=\frac{1}{\left|z_{0}\right|_{ \pm}^{2}}\left[z_{0}^{i} \delta_{k l}^{ \pm}-\frac{z_{0}^{r}}{2}\left(\delta_{k}^{i} \delta_{l r}^{ \pm}+\delta_{l}^{i} \delta_{k r}^{ \pm}\right)\right]
$$

and thus to the new variable

$$
\eta^{i}=z^{i}+\frac{\epsilon}{2\left|z_{0}\right|_{ \pm}^{2}}\left(z_{0}^{i}|z|_{ \pm}^{2}-z^{i}\left\langle z, z_{0}\right\rangle_{ \pm}\right) .
$$

Note that the change of variable is such that

$$
|\eta|_{ \pm}^{2}=|z|_{ \pm}^{2}+O\left(\epsilon^{2}\right) .
$$

With the variable $\eta$, the weakly nonlinear dynamics of the disturbance is generated by the Poisson matrix (3.1). The corresponding Casimir function is

$$
\left\langle z_{0}, \eta\right\rangle_{ \pm}=\left\langle z_{0}, z\right\rangle_{ \pm}+\frac{\epsilon}{2}|z|_{ \pm}^{2}-\frac{\epsilon}{2\left|z_{0}\right|_{ \pm}^{2}}\left\langle z_{0}, z\right\rangle_{ \pm}^{2} .
$$

The first two terms can be recognized as the exact Casimir $\epsilon^{-1}\left(\left|z_{0}+\epsilon z\right|_{ \pm}^{2}-\left|z_{0}\right|_{ \pm}^{2}\right) / 2$. The time derivative of the third term is $O\left(\epsilon^{2}\right)$, so that it appears constant to the order of accuracy considered here.

\section{$3.2 \quad$ Heavy-top bracket}

Another simple bracket is that of the heavy top for which the dynamical variables consist of the angular momentum $\mu$ and the direction of the gravity field $\gamma: z=(\mu, \gamma)^{\mathrm{T}}$. The Poisson matrix defining this bracket can be written

$$
J^{i j}=\left(\begin{array}{cc}
-\varepsilon_{i j k} \mu^{k} & -\varepsilon_{i j k} \gamma^{k} \\
-\varepsilon_{i j k} \gamma^{k} & 0
\end{array}\right) .
$$


The heavy top bracket [22] has a Lie-Poisson structure based on a Lie algebra extension of $\mathfrak{s o}(3)$, but the corresponding Lie algebra is not semisimple [25]; we will thus use (2.22) to find the variable transformation (2.9). For simplicity, we restrict our attention to equilibrium solutions with $\mu=$ $\left(0,0, M_{3}\right)$ and $\gamma=(0,0,1)$ which arise for the Lagrange top (top with a symmetry). The following tensors are required for $(2.22)$ and can be easily obtained:

$$
J_{0}^{i j}=\left(\begin{array}{cc}
-M_{3} \varepsilon_{i j 3} & -\varepsilon_{i j 3} \\
-\varepsilon_{i j 3} & 0
\end{array}\right), \chi_{i}^{1}=\left(\begin{array}{c}
\delta_{i 3} \\
0
\end{array}\right), \chi_{i}^{2}=\left(\begin{array}{c}
0 \\
\delta_{i 3}
\end{array}\right), \omega_{n k}=-\frac{1}{3}\left(\begin{array}{cc}
0 & \varepsilon_{n k 3} \\
\varepsilon_{n k 3} & -M_{3} \varepsilon_{n k 3}
\end{array}\right) .
$$

Because the null eigenvectors are orthonormal, we have taken $\chi_{\alpha}^{i}=\chi_{i}^{\alpha}$. A convenient way to denote tensors such as $D^{i}{ }_{k l}$ is to make the separation $\left(D^{\bar{\imath}}{ }_{k l}, D^{\hat{\imath}}{ }_{k l}\right)$ between the components $i$ corresponding to $\mu$ (denoted by $\bar{\imath}$ ) and those corresponding to $\gamma$ (denoted by $\hat{\imath}$ ). With this convention, we can write $\partial J_{0}^{i n} / \partial z_{0}^{m}$ as the two triplets of $6 \times 6$ matrices

$$
\frac{\partial J_{0}^{\bar{\imath} n}}{\partial z_{0}^{m}}=\left(\begin{array}{cc}
-\varepsilon_{\bar{i} n m} & 0 \\
0 & -\varepsilon_{\bar{\imath} n m}
\end{array}\right), \quad \frac{\partial J_{0}^{\hat{\imath} n}}{\partial z_{0}^{m}}=\left(\begin{array}{cc}
0 & -\varepsilon_{\hat{\imath} n m} \\
0 & 0
\end{array}\right), \quad \bar{\imath}, \hat{\imath}=1,2,3,
$$

where $n(m)$ is the row (column) index. Noting that the general expression for two orthonormal null eigenvectors of $J^{i j}$ is

$$
\chi_{k}^{1}=\frac{1}{N}\left(\gamma^{k}, \mu^{k}-(\mu \cdot \gamma) /|\gamma|^{2} \gamma^{k}\right)^{\mathrm{T}}, \quad \chi_{k}^{2}=\frac{1}{|\gamma|}\left(0, \gamma^{k}\right)^{\mathrm{T}},
$$

where $N^{2}:=|\mu|^{2}+|\gamma|^{2}-(\mu \cdot \gamma)^{2} /|\gamma|^{2}$, one can calculate the two matrices

$$
\begin{aligned}
\frac{\partial \chi_{k}^{1}}{\partial z_{0}^{m}} & =\left(\begin{array}{cc}
0 & \delta_{k m}-\delta_{k 3} \delta_{m 3} \\
\delta_{k m}-\delta_{k 3} \delta_{m 3} & -M_{3}\left(\delta_{k m}-\delta_{k 3} \delta_{m 3}\right)
\end{array}\right) \\
\frac{\partial \chi_{k}^{2}}{\partial z_{0}^{m}} & =\left(\begin{array}{cc}
0 & 0 \\
0 & \left.\delta_{k m}-\delta_{k 3} \delta_{m 3}\right)
\end{array}\right)
\end{aligned}
$$

With these results, and using the skew-symmetry of $\omega_{n k}$, one can compute (2.22) through simple matrix multiplications and additions. After simplifications, one finds

$$
\begin{aligned}
D^{\bar{\imath}}{ }_{k l} & =\left(\begin{array}{cc}
0 & \delta_{k l} \delta_{\bar{\imath} 3}-\frac{1}{2}\left(\delta_{\bar{\imath} k} \delta_{l 3}+\delta_{\bar{\imath} l} \delta_{k 3}\right) \\
\delta_{k l} \delta_{\bar{\imath} 3}-\frac{1}{2}\left(\delta_{\bar{\imath} k} \delta_{l 3}+\delta_{\bar{\imath} l} \delta_{k 3}\right) & -M_{3}\left[\delta_{k l} \delta_{\bar{\imath} 3}-\frac{1}{2}\left(\delta_{\bar{\imath} k} \delta_{l 3}+\delta_{\bar{\imath} l} \delta_{k 3}\right)\right]
\end{array}\right) \\
D^{\hat{\imath}}{ }_{k l} & =\left(\begin{array}{cc}
0 & 0 \\
0 & \delta_{k l} \delta_{\hat{\imath} 3}-\frac{1}{2}\left(\delta_{\hat{\imath} k} \delta_{l 3}+\delta_{\hat{\imath} l} \delta_{k 3}\right)
\end{array}\right) .
\end{aligned}
$$

The new variables which can be used to derive the Hamiltonian equations for the weakly nonlinear dynamics of the heavy top take thus the form

$$
\left\{\begin{array}{rl}
\eta^{\alpha} & =\mu^{1}-\frac{\epsilon}{2}\left(\mu^{\alpha} \gamma^{3}+\mu^{3} \gamma^{\alpha}-M_{3} \gamma^{\alpha} \gamma^{3}\right) \\
\eta^{3} & =\mu^{3}+\frac{\epsilon}{2}\left\{\mu^{1} \gamma^{1}+\mu^{2} \gamma^{2}-M_{3}\left[\left(\gamma^{1}\right)^{2}+\left(\gamma^{2}\right)^{2}\right]\right\} \\
\sigma^{\alpha} & =\gamma^{1}-\frac{\epsilon}{2} \gamma^{\alpha} \gamma^{3} \\
\sigma^{3} & =\sigma^{3}+\frac{\epsilon}{2}\left[\left(\gamma^{1}\right)^{2}+\left(\gamma^{2}\right)^{2}\right]
\end{array} \quad \alpha=1,2\right.
$$

\subsection{Two-spin system}

To illustrate the interest of the approach, we now consider the specific example of a system constituted of two coupled spins in a magnetic field. This system is defined by the Hamiltonian

$$
H=B \cdot \omega_{(1)}+B \cdot \omega_{(2)}+a \omega_{(1)} \cdot \omega_{(2)},
$$

where $B$ is the external magnetic field, $a$ a coupling coefficient, and $\omega_{(1)}$ and $\omega_{(2)}$ the angular momentum vectors of the two spins, and by the bracket

$$
\{f, g\}=\sum_{\alpha=1}^{2} \varepsilon_{i j k} \omega_{(\alpha)}^{k} \frac{\partial f}{\partial \omega_{(\alpha)}^{i}} \frac{\partial g}{\partial \omega_{(\alpha)}^{j}} .
$$


i.e. the sum of two rigid body brackets corresponding to each spin. The dynamical equations are

$$
\dot{\omega}_{(\alpha)}=B \times \omega_{(\alpha)}+a \omega_{(\alpha+1)} \times \omega_{(\alpha)}, \quad \alpha=1,2 .
$$

(The system is integrable, because $\omega_{(1)} \cdot \omega_{(2)}$ is conserved in addition to the energy and two Casimirs.) An obvious equilibrium solution of these equations is given by

$$
\omega_{(1)_{0}}=\omega_{(2)_{0}}=m B .
$$

Noting that the bracket possesses two Casimir functions

$$
C^{\alpha}=\left|\omega_{(\alpha)}\right|^{2}, \quad \alpha=1,2,
$$

it is easily seen that this equilibrium is a critical point of

$$
F=H+\lambda_{1} C^{1}+\lambda_{2} C^{2}
$$

with

$$
\lambda_{1}=\lambda_{2}=\lambda:=-\frac{1+a m}{2 m} .
$$

We can choose one coordinate axis aligned with the external magnetic field, so that $B=\left(B_{1}, 0,0\right)$ and $\omega_{(1)_{0}}=\omega_{(2)_{0}}=\left(m B_{1}, 0,0\right)$. We now study the weakly nonlinear evolution of a small disturbance to this equilibrium. We therefore introduce the decomposition

$$
\omega_{(\alpha)}=\omega_{(\alpha)_{0}}+\epsilon z_{(\alpha)}, \quad \alpha=1,2, \quad \text { with } \epsilon \ll 1 .
$$

In terms of the perturbation $z_{(\alpha)}$, the free energy $(2.3)$ is given by

$$
F_{z}:=\epsilon^{-2}\left[F\left(\omega_{(\alpha)_{0}}+\epsilon z_{(\alpha)}\right)-F\left(\omega_{(\alpha)_{0}}\right)\right]=a z_{(1)} \cdot z_{(2)}+\lambda\left(\left|z_{(1)}\right|^{2}+\left|z_{(2)}\right|^{2}\right),
$$

which is exactly quadratic. The nonlinearity in the equation governing the evolution of $z_{(\alpha)}$ comes thus entirely from the bracket. Note that the stability of the equilibrium can be tested using Dirichlet's criterion. The matrix

$$
\frac{1}{2} \frac{\partial^{2} F_{z}}{\partial z_{(1)} \partial z_{(2)}}=\left(\begin{array}{cc}
\lambda & a / 2 \\
a / 2 & \lambda
\end{array}\right)
$$

has the eigenvalues $-(1+2 a m) / 2 m$ and $-1 / 2 m$ and is thus sign-definite if $1+2 a m>0$. This condition ensures the nonlinear stability of the equilibrium.

Since the nonlinear bracket is the combination of two rigid body brackets, we can apply the change of variable (3.5) with $z_{0}=\left(m B_{1}, 0,0\right)^{\mathrm{T}}$ and define

$$
\left\{\begin{array}{l}
\eta_{(\alpha)}^{1}=z_{(\alpha)}^{1}+\frac{\epsilon}{2 m B_{1}}\left[\left(z_{(\alpha)}^{2}\right)^{2}+\left(z_{(\alpha)}^{3}\right)^{2}\right] \\
\eta_{(\alpha)}^{2}=z_{(\alpha)}^{2}-\frac{\epsilon}{2 m B_{1}} z_{(\alpha)}^{1} z_{(\alpha)}^{2} \\
\eta_{(\alpha)}^{3}=z_{(\alpha)}^{3}-\frac{\epsilon}{2 m B_{1}} z_{(\alpha)}^{1} z_{(\alpha)}^{3}
\end{array} \quad \alpha=1,2\right.
$$

In accordance with (2.7), the dynamics of $\eta_{(\alpha)}$ including the $O(\epsilon)$ nonlinear terms is determined by the constant bracket

$$
\{f, g\}_{0}=m B_{1} \sum_{\alpha=1}^{2} \varepsilon_{i j 1} \frac{\partial f}{\partial \eta_{(\alpha)}^{i}} \frac{\partial g}{\partial \eta_{(\alpha)}^{j}} .
$$

It is therefore immediately clear that

$$
\dot{\eta}_{(1)}^{1}=\dot{\eta}_{(2)}^{1}=0, \quad \text { hence } \eta_{(1)}^{1}=c_{1}, \eta_{(2)}^{1}=c_{2},
$$

where $c_{1}, c_{2}$ are fixed by the initial conditions. In fact, $\eta_{(1)}^{1}$ and $\eta_{(2)}^{1}$ are the Casimir functions of the system. 
The nonlinearity in the evolution equations for $\eta_{(\alpha)}$ appears as cubic terms in the Hamiltonian. To compute those terms, we need to invert the transformation (3.11) according to

$$
\left\{\begin{array}{rl}
z_{(\alpha)}^{1} & =\eta_{(\alpha)}^{1}-\frac{\epsilon}{2 m B_{1}}\left[\left(\eta_{(\alpha)}^{2}\right)^{2}+\left(\eta_{(\alpha)}^{3}\right)^{2}\right]+O\left(\epsilon^{2}\right) \\
z_{(\alpha)}^{2} & =\eta_{(\alpha)}^{2}+\frac{\epsilon}{2 m B_{1}} \eta_{(\alpha)}^{1} \eta_{(\alpha)}^{2}+O\left(\epsilon^{2}\right) \\
z_{(\alpha)}^{3} & =\eta_{(\alpha)}^{3}+\frac{\epsilon}{2 m B_{1}} \eta_{(\alpha)}^{1} \eta_{(\alpha)}^{3}+O\left(\epsilon^{2}\right)
\end{array} \quad \alpha=1,2\right.
$$

Introducing this into the Hamiltonian (3.10) and using (3.6) yields

$$
F_{\eta}=a \eta_{(1)} \cdot \eta_{(2)}+\lambda\left(\left|\eta_{(1)}\right|^{2}+\left|\eta_{(2)}\right|^{2}\right)+\frac{\epsilon a}{2 m B_{1}} \sum_{i=2}^{3}\left(\eta_{(1)}^{i}-\eta_{(2)}^{i}\right)\left(\eta_{(1)}^{1} \eta_{(2)}^{i}-\eta_{(2)}^{1} \eta_{(1)}^{i}\right)+O\left(\epsilon^{2}\right)
$$

The weakly nonlinear equations for $\eta:=\left(\eta_{(1)}^{2}, \eta_{(1)}^{3}, \eta_{(2)}^{2}, \eta_{(2)}^{3}\right)^{\mathrm{T}}$ can then be rendered canonical. By construction, the necessary variable transformation uses the action-angle coordinates $\left(J_{(\alpha)}, \theta_{(\alpha)}\right)$ which make the linearized equations for $z$ canonical; these coordinates are derived in Appendix A. Applied to $\eta_{\alpha}$, they are defined by

$$
\left\{\begin{array}{l}
\eta_{(1)}^{2}=\sqrt{\left|m B_{1}\right|}\left[-\sqrt{J_{(1)}} \sin \left(s \theta_{(1)}\right)+\sqrt{J_{(2)}} \sin \left(s \theta_{(2)}\right)\right] \\
\left.\eta_{(1)}^{3}=\sqrt{\left|m B_{1}\right|}-\sqrt{J_{(1)}} \cos \left(s \theta_{(1)}\right)+\sqrt{J_{(2)}} \cos \left(s \theta_{(2)}\right)\right] \\
\eta_{(2)}^{2}=\sqrt{\left|m B_{1}\right|}\left[\sqrt{J_{(1)}} \sin \left(s \theta_{(1)}\right)+\sqrt{J_{(2)}} \sin \left(s \theta_{(2)}\right)\right] \\
\eta_{(2)}^{3}=\sqrt{\left|m B_{1}\right|}\left[\sqrt{J_{(1)}} \cos \left(s \theta_{(1)}\right)+\sqrt{J_{(2)}} \cos \left(s \theta_{(2)}\right)\right]
\end{array},\right.
$$

where $s:=\operatorname{sign}\left(m B_{1}\right)$. In terms of $\left(J_{(\alpha)}, \theta_{(\alpha)}\right)$, the Hamiltonian (3.15) becomes

$$
\begin{aligned}
F_{\left(J_{(\alpha)}, \theta_{(\alpha)}\right)} & =-s \sum_{\alpha=1}^{2} \sigma_{(\alpha)} J_{(\alpha)}+a c_{1} c_{2} \\
& -\epsilon \operatorname{sa} \sqrt{J_{(1)}}\left[\left(c_{1}+c_{2}\right) \sqrt{J_{(1)}}+\left(c_{1}-c_{2}\right) \sqrt{J_{(2)}} \cos \left(\theta_{(1)}-\theta_{(2)}\right)\right]+O\left(\epsilon^{2}\right),
\end{aligned}
$$

where

$$
\sigma_{(1)}:=B_{1}(1+2 a m) \text { and } \sigma_{(2)}:=B_{1} .
$$

This completes the reformulation of the weakly nonlinear dynamics of the two-spin system as a canonical system to which standard pertubation methods can be applied. As an immediate illustration, we can read off from (3.16) the frequencies $\sigma_{(1)}+\epsilon a\left(c_{1}+c_{2}\right)$ and $\sigma_{(2)}$ and interpret the correction $\epsilon a\left(c_{1}+c_{2}\right)$ to $\sigma_{(1)}$ as a nonlinear frequency shift. Further details on the perturbation theory are given in Appendix A.

\section{Infinite-dimensional systems}

The extension of (1.2) to partial differential equations is

$$
\frac{\partial \zeta}{\partial t}=\mathrm{J} \frac{\delta \mathcal{H}}{\delta \zeta},
$$

where $\zeta$ is the vector of the dynamical variables, $\mathrm{J}$ is a skew-adjoint operator that we call the Poisson operator, and the Hamiltonian $\mathcal{H}$ is a functional

$$
\mathcal{H}=\int H(x, t, \zeta, \nabla \zeta, \ldots) \mathrm{d} \mathbf{x} .
$$

The Jacobi identity satisfied by $\mathrm{J}$ is best written in terms of the Poisson bracket

$$
\{\mathcal{F}, \mathcal{G}\}:=\int \frac{\delta \mathcal{F}}{\delta \zeta} \mathrm{J}(\zeta) \frac{\delta \mathcal{G}}{\delta \zeta} \mathrm{d} \mathbf{x}
$$




$$
\{\mathcal{F},\{\mathcal{G}, \mathcal{H}\}\}+\{\mathcal{G},\{\mathcal{H}, \mathcal{F}\}\}+\{\mathcal{H},\{\mathcal{F}, \mathcal{G}\}\}=0 .
$$

As in the finite-dimensional case, the operator $J$ can be degenerate and there exists Casimir functionals $\mathcal{C}$ such that

$$
\{\mathcal{F}, \mathcal{C}\}=0, \quad \forall \mathcal{F} .
$$

Consider an equilibrium $\zeta_{0}$ of (4.1). Usually only trivial equilibria are critical points of $\mathcal{H}$, while equilibria of interest are critical points of the combination

$$
\mathcal{F}[\zeta]:=\mathcal{H}[\zeta]+\mathcal{C}[\zeta]
$$

for a well-chosen Casimir functional, i.e.

$$
\mathrm{J}_{0} \frac{\delta \mathcal{F}_{0}}{\delta \zeta_{0}}:=\mathrm{J}\left(\zeta_{0}\right) \frac{\delta \mathcal{F}\left[\zeta_{0}\right]}{\delta \zeta_{0}}=0 .
$$

To study the evolution of a small disturbance to $\zeta_{0}$, we introduce the decomposition

$$
\zeta=\zeta_{0}+\epsilon \zeta^{\prime}
$$

and omit the prime. The disturbance obeys the equation

$$
\frac{\partial \zeta}{\partial t}=\mathrm{J}_{\zeta} \frac{\delta \mathcal{F}_{\zeta}}{\delta \zeta}
$$

where

$$
\mathrm{J}_{\zeta}:=\mathrm{J}\left(\zeta_{0}+\epsilon \zeta\right) \quad \text { and } \quad \mathcal{F}_{\zeta}:=\epsilon^{-2}\left[F\left(\zeta_{0}+\epsilon \zeta\right)-F\left(\zeta_{0}\right)\right] .
$$

Because of (4.2), the linearized equation given by

$$
\frac{\partial \zeta}{\partial t}=\left.\mathrm{J}_{0} \frac{\delta^{2} \mathcal{F}_{\zeta}}{\delta \zeta^{2}}\right|_{\zeta=0} \zeta
$$

is Hamiltonian. An equation describing the weakly nonlinear evolution of the disturbance can be obtained by truncating (4.4) to $O(\epsilon)$. Generally, it will not be Hamiltonian, because of the truncation of $J_{\zeta}$. To find a truncation which is Hamiltonian, we follow the same idea as for finite-dimensional systems: we seek a near-identity transformation from $\zeta$ to $\eta$ such that

$$
\mathrm{J}_{\eta}=\mathrm{J}_{0}+O\left(\epsilon^{2}\right),
$$

where $J_{\eta}$ is the Poisson operator for $\eta$. The weakly nonlinear evolution can therefore be determined from the Hamiltonian equation

$$
\frac{\partial \eta}{\partial t}=\mathrm{J}_{0} \frac{\delta \mathcal{F}_{\eta}}{\delta \eta}+O\left(\epsilon^{2}\right)
$$

where $\mathcal{F}_{\eta}:=\mathcal{F}_{\zeta}[\zeta(\eta)]$ can be truncated to $O(\epsilon)$. We emphasize that this procedure does not modify the independent variables used; this is important for practical use of the weakly nonlinear equations (cf. Lagrangian vs. Eulerian description).

The required change of variable has the general form

$$
\eta=\zeta+\epsilon \mathrm{O}(\zeta ; \zeta)
$$

where $\mathrm{O}$ is a symmetric bilinear operator to be determined. From this, the operator $\mathrm{J}_{\eta}$, defined by

$$
\{\mathcal{F}, \mathcal{G}\}=\int \frac{\delta \mathcal{F}}{\delta \eta} \mathrm{J}_{\eta} \frac{\delta \mathcal{G}}{\delta \eta} \mathrm{d} \mathbf{x}=\int \frac{\delta \mathcal{F}}{\delta \zeta} \mathrm{J}_{\zeta} \frac{\delta \mathcal{G}}{\delta \zeta} \mathrm{d} \mathbf{x},
$$

can be obtained, and an equation for $\mathrm{O}$ can be found from condition (4.5). Although this procedure is general, it is clear in light of the finite-dimensional case that the algebra involved can be quite complex. Therefore, we shall consider particular examples to show how the change of variable can be found and exploited. Our examples concern two different brackets and (at least) four different physical systems: (a) Euler bracket [4, 26, 27], which appears for the two-dimensional scalar vortex dynamics (including quasi-geostrophy) and for the one-dimensional Vlasov-Poisson equation [28], (b) the bracket which appears for two-dimensional stratified fluid and for two-dimensional MHD $[29,30]$. In all cases, we will consider simple equlibria, depending on one coordinate only. 


\section{Euler bracket, vortex dynamics and Vlasov-Poisson equa- tions}

The Euler bracket in two dimensions $(x, y)$ can be written

$$
\{\mathcal{F}, \mathcal{G}\}=\iint \zeta\left[\frac{\delta \mathcal{F}}{\delta \zeta}, \frac{\delta \mathcal{G}}{\delta \zeta}\right] \mathrm{d} x \mathrm{~d} y
$$

where

$$
[f, g]:=\frac{\partial f}{\partial x} \frac{\partial g}{\partial y}-\frac{\partial f}{\partial y} \frac{\partial g}{\partial x}
$$

and corresponds to the Poisson operator

$$
\mathrm{J}(\zeta)=-[\zeta, \cdot]
$$

The equilibrium solution is assumed to be a function of $y$ only, $\zeta_{0}=\zeta_{0}(y)$, and therefore,

$$
\mathrm{J}_{0}=\zeta_{0}^{\prime} \frac{\partial}{\partial x}
$$

with $\zeta_{0}^{\prime}:=\mathrm{d} \zeta_{0} / \mathrm{d} y$. We consider a simple form of the change of variable (4.6) given by

$$
\eta=\zeta+\frac{\epsilon}{2} \mathrm{D} \zeta^{2}
$$

where the linear operator $\mathrm{D}=\mathrm{D}\left(\zeta_{0}\right)$ can be expected to involve $\partial_{y}$ and not $\partial_{x}$. The functional derivatives with respect to $\zeta$ and $\eta$ are related by

$$
\frac{\delta F}{\delta \zeta}=\left(1+\epsilon \zeta \mathrm{D}^{\dagger}\right) \frac{\delta F}{\delta \eta},
$$

where $^{\dagger}$ denotes the adjoint. From (4.7) it is then found that

$$
\begin{aligned}
\iint \frac{\delta \mathcal{F}}{\delta \zeta} \mathrm{J}_{\eta} \frac{\delta \mathcal{G}}{\delta \zeta} \mathrm{d} x \mathrm{~d} y & =\iint\left\{\frac{\delta \mathcal{F}}{\delta \zeta} \mathrm{J}_{0} \frac{\delta \mathcal{G}}{\delta \zeta}+\epsilon \zeta\left(\zeta_{0}^{\prime} \mathrm{D}^{\dagger}\left(\frac{\delta \mathcal{F}}{\delta \zeta}\right) \partial_{x}\left(\frac{\delta \mathcal{G}}{\delta \zeta}\right)\right.\right. \\
& \left.\left.-\zeta_{0}^{\prime} \partial_{x}\left(\frac{\delta \mathcal{F}}{\delta \zeta}\right) \mathrm{D}^{\dagger}\left(\frac{\delta \mathcal{G}}{\delta \zeta}\right)+\left[\frac{\delta \mathcal{F}}{\delta \zeta}, \frac{\delta \mathcal{G}}{\delta \zeta}\right]\right)\right\} \mathrm{d} x \mathrm{~d} y+O\left(\epsilon^{2}\right)
\end{aligned}
$$

Clearly, condition (4.5) is satisfied if

$$
\mathrm{D}^{\dagger}=\frac{1}{\zeta_{0}^{\prime}} \frac{\partial}{\partial y} \quad \text { i.e. } \quad \mathrm{D}=-\frac{\partial}{\partial y} \frac{1}{\zeta_{0}^{\prime}} .
$$

Hence, the change of variable sought is

$$
\eta=\zeta-\epsilon \frac{\partial}{\partial y}\left(\frac{\zeta^{2}}{2 \zeta_{0}^{\prime}}\right) .
$$

For now, let us assume that $\zeta_{0}^{\prime} \neq 0$. The inverse transformation is given by

$$
\zeta=\eta+\epsilon \frac{\partial}{\partial y}\left(\frac{\eta^{2}}{2 \zeta_{0}^{\prime}}\right)+O\left(\epsilon^{2}\right) .
$$

Note that the use of $\eta$ requires not only $\epsilon \ll 1$, but also

$$
\epsilon \frac{\partial \zeta}{\partial y} \ll \zeta_{0}^{\prime}
$$

(and similar conditions for higher-order derivatives).

We make a few remarks to relate the transformation (5.4)-(5.5) to previous results. 
1. It is clear from the bracket (5.2) for $\eta$ that

$$
\frac{\partial \bar{\eta}}{\partial t}=O\left(\epsilon^{2}\right)
$$

where $^{-}$denotes the average in the $x$ direction; therefore,

$$
\bar{\zeta}=\epsilon \frac{\partial}{\partial y}\left(\frac{\overline{\zeta^{2}}}{2 \zeta_{0}^{\prime}}\right)+C(y)+O\left(\epsilon^{2}\right)=\epsilon \frac{\partial}{\partial y}\left(\frac{\overline{\hat{\zeta}^{2}}}{2 \zeta_{0}^{\prime}}\right)+C(y)+O\left(\epsilon^{2}\right),
$$

where $\hat{\zeta}:=\zeta-\bar{\zeta}$ is the wavy part of the disturbance, and $C(y)$ is fixed by the initial conditions. That the mean value of $\zeta$ can be evaluated to leading order knowing the wavy part of the disturbance only is a standard result of wave-mean flow interaction theory for Euler or quasigeostrophic equations (e.g. [31]). It is often formulated using the velocity in the $x$ direction defined by

$$
u:=\int \zeta \mathrm{d} y
$$

as

$$
\frac{\partial \bar{u}}{\partial t}=\epsilon \frac{\overline{\hat{\zeta}^{2}}}{2 \zeta_{0}^{\prime}}+O\left(\epsilon^{2}\right)
$$

and the quantity $\zeta^{2} / 2 \zeta_{0}^{\prime}$ is often refered to as the pseudomomentum (up to sign) [32], but can be traced back to the physical monentum of the fluid [33]. Here, we relate this result to the Poisson bracket, independently of the Hamiltonian.

2. The approximate constancy of $\bar{\eta}$, which results from the imposed Poisson bracket, suggests that $\eta$ must be given by the old variable minus their $x$-average (compare (5.4) and (5.8)). This yields a procedure for constructing the change of variable if we can compute a priori this average, i.e. the right-hand side of (5.8). This is possible using the concept of dynamical accessibility [34]. This expresses the fact the perturbed vorticity $\zeta_{0}+\epsilon \zeta$ lies on the same symplectic leaf as the equilibrium vorticity $\zeta_{0}$, that is, the values of the Casimir functionals for the perturbed flows are the same as the equilibrium values. This can generally be arranged by an appropriate definition of the equilibrium flow. The dynamical accessibility of $\zeta_{0}+\varepsilon \zeta$ can be written explicitly in the form

$$
\epsilon \zeta=(\exp \{-\mathcal{G}, \cdot\}-1) \zeta_{0}=(\exp [\epsilon g, \cdot]-1) \zeta_{0}=\epsilon g_{x} \zeta_{0}^{\prime}+\frac{1}{2} \epsilon^{2}\left[g, g_{x} \zeta_{0}^{\prime}\right]+\ldots,
$$

where $g$ is an arbitrary function and

$$
\mathcal{G}:=\epsilon \iint g \zeta \mathrm{d} x \mathrm{~d} y .
$$

Applying ${ }^{-}$and noting that $g_{x}=\zeta / \zeta_{0}^{\prime}+O(\epsilon)$, one finds (5.8) and hence an indication of the change of variable (5.4). Note that the presence/absence of ${ }^{-}$is not really relevant, as one can add a term in $\partial_{x}$ to (5.4) without changing the bracket to the order of accuracy considered.

3. The dynamical accessibility condition (5.9) also make clear that the variable transformation (5.4) is in fact well defined even when $\zeta_{0}^{\prime}=0$ at some $y$.

4. The transformation (5.4) is the related Zakharov-Piterbarg variable transformation [35] for the quasi-geostrophic system. Considering the particular case $\zeta_{0}=\beta y$, they found that the new variable implicitly defined by

$$
\eta_{\mathrm{ZP}}(x, y)=\zeta\left[x, y-\epsilon \beta^{-1} \eta_{\mathrm{ZP}}(x, y)\right]
$$

is such that the Poisson operator is given by

$$
\mathrm{J}_{\eta_{\mathrm{ZP}}}=\zeta_{0}^{\prime} \frac{\partial}{\partial x}=\beta \frac{\partial}{\partial x}
$$


to all orders in $\epsilon$. Expanding (5.10) in $\epsilon$ and comparing with (5.4) it is clear that our transformation is the first-order approximation to that of Zakharov and Piterbarg. In fact, their transformation can be extended to more general forms of $\zeta_{0}$. Suppose that $\zeta_{0}(y)$ is monotonic and admits the inverse $y_{0}(\zeta)$, and consider the transformation of both dependent and independent variables $(x, y, \zeta) \rightarrow\left(x, z, \eta_{\mathrm{C}}\right)$ defined by

$$
z=y_{0}\left[\zeta_{0}(y)+\epsilon \zeta(x, y)\right] \quad \text { and } \quad \eta_{\mathrm{C}}=\eta_{\mathrm{C}}[x, y, \zeta]=\left.\epsilon^{-1} \frac{\mathrm{d} \zeta_{0}}{\mathrm{~d} y_{0}}\right|_{z}(z-y)
$$

Assuming that this transformation is one to one (level sets of $\zeta_{0}+\epsilon \zeta$ remain graphs over $x$ ), it can be shown that the Poisson operator for $\eta_{\mathrm{C}}$ is exactly given by (5.2). This is detailed in Appendix B, where we also show that $\eta_{\mathrm{C}}$ reduce to $\eta_{\mathrm{ZP}}$ for $\zeta_{0}=\beta y$ and that $\eta$ defined by (5.4) is simply the $O(\epsilon)$ approximation to $\eta_{\mathrm{C}}$. It is interesting to note the "Lagrangian character" of the transformed variables: $z$ is in fact a vorticity (transformed in a space coordinate through the function $y_{0}$ ), hence a label, and $\eta_{\mathrm{C}}$ is related to a displacement.

Below, we consider the vortex dynamics equation and the one-dimensional Vlasov-Poisson equation and use the inverse transformation (5.5) to compute the Hamiltonian and derive transformed evolution equations.

\subsection{Vortex dynamics equation}

The two-dimensional vorticity equation for incompressible or quasi-geostrophic flows is obtained using the bracket (5.1) with the Hamiltonian [32, 36]

$$
\mathcal{H}=\frac{1}{2} \iint\left(|\nabla \psi|^{2}+R^{-2} \psi^{2}\right) \mathrm{d} x \mathrm{~d} y .
$$

Here, $\psi$ is the streamfunction, related to the vorticity $\zeta$ through

$$
\zeta=\nabla^{2} \psi-R^{-2} \psi
$$

where the second term takes into account the effect of a free surface in the quasi-geostrophic approximation, $R$ being the Rossby radius of deformation. The evolution equation reads

$$
\zeta_{t}+[\psi, \zeta]=0 .
$$

A basic equilibrium consists of a parallel shear flow $U(y)$ superposed to the basic planetary rotation:

$$
\zeta_{0}=f_{0}+\beta y-U^{\prime}
$$

Introducing the decomposition (4.3), one finds the equation for the disturbance

$$
\zeta_{t}+U \zeta_{x}+\zeta_{0}^{\prime} \psi_{x}+\epsilon[\psi, \zeta]=0
$$

Let us compute the free energy (pseudoenergy) (4.4) using the Casimirs functionals which have the form

$$
\mathcal{C}=\iint C\left(\zeta_{0}+\epsilon \zeta\right) \mathrm{d} x \mathrm{~d} y
$$

for any function $C(\cdot)$. It is well known that the proper choice of $C(\cdot)$ is

$$
C(\cdot)=\int_{\zeta_{0}}^{(\cdot)} \psi_{0}(\mu) \mathrm{d} \mu
$$

where $\psi_{0}$ denotes the functional relationship between the streamfunction and the vorticity which necessarily exists for equilibrium solutions. The free energy is thus given by

$$
\mathcal{F}_{\zeta}=\iint\left\{\left[\frac{1}{2}|\nabla \psi|^{2}+R^{-2} \psi^{2}\right]+\epsilon^{-1} \int_{0}^{\zeta}\left[\psi_{0}\left(\zeta_{0}+\epsilon \mu\right)-\psi_{0}\left(\zeta_{0}\right)\right] \mathrm{d} \mu\right\} \mathrm{d} x \mathrm{~d} y .
$$


As only the terms up to $O(\epsilon)$ are required for our purpose, we write

$$
\mathcal{F}_{\zeta}=\frac{1}{2} \iint\left[|\nabla \psi|^{2}+R^{-2} \psi^{2}-\frac{U}{\zeta_{0}^{\prime}} \zeta^{2}-\frac{\epsilon}{3 \zeta_{0}^{\prime}} \frac{\mathrm{d}}{\mathrm{d} y}\left(\frac{U}{\zeta_{0}^{\prime}}\right) \zeta^{3}\right] \mathrm{d} x \mathrm{~d} y+O\left(\epsilon^{2}\right) .
$$

We now introduce (5.5) into (5.14). Because the relationship between $\psi$ and $\eta$ is nonlinear, we define the two fields $\varphi$ and $\vartheta$ by

$$
\nabla^{2} \varphi-R^{-2} \varphi=\eta \quad \text { and } \quad \nabla^{2} \vartheta-R^{-2} \vartheta=\frac{1}{2} \frac{\partial}{\partial y}\left(\frac{\eta^{2}}{2 \zeta_{0}^{\prime}}\right)
$$

so that $\psi=\varphi+\epsilon \vartheta$ and thus

$$
|\nabla \psi|^{2}+R^{-2} \psi^{2}=|\nabla \varphi|^{2}+R^{-2} \varphi^{2}+2 \epsilon \nabla \varphi \cdot \nabla \vartheta+2 \epsilon R^{-2} \varphi \vartheta+O\left(\epsilon^{2}\right) .
$$

The two other terms in (5.14) require a little more calculation (with some integrations by parts). Truncating to $O(\epsilon)$, it is finally found that

$$
\mathcal{F}_{\eta}=\frac{1}{2} \iint\left(|\nabla \varphi|^{2}+R^{-2} \varphi^{2}-\frac{U}{\zeta_{0}^{\prime}} \eta^{2}+2 \epsilon \nabla \varphi \cdot \nabla \vartheta+2 \epsilon R^{-2} \varphi \vartheta+\epsilon \frac{U^{\prime}}{3{\zeta_{0}^{\prime}}^{2}} \eta^{3}\right) \mathrm{d} x \mathrm{~d} y .
$$

Taking into account (5.15), it is easy to calculate the functional derivative of $\mathcal{F}_{\eta}$ :

$$
\frac{\delta \mathcal{F}_{\eta}}{\delta \eta}=-\varphi-\frac{U}{\zeta_{0}^{\prime}} \eta-\epsilon \vartheta+\epsilon \frac{1}{\zeta_{0}^{\prime}} \varphi_{y} \eta+\epsilon \frac{U^{\prime}}{2{\zeta_{0}^{\prime}}^{2}} \eta^{2} .
$$

The evolution equation for $\eta$ is then found using the Poisson operator (5.2); it reads

$$
\eta_{t}=-U \eta_{x}-\zeta_{0}^{\prime} \varphi_{x}+\epsilon\left(-\zeta_{0}^{\prime} \vartheta_{x}+\varphi_{x y} \eta+\varphi_{y} \eta_{x}+\frac{U^{\prime}}{\zeta_{0}^{\prime}} \eta \eta_{x}\right) .
$$

That this equation is a truncated version of (5.12) can be verified by taking the time derivative of (5.4), using (5.12) and (5.5), and neglecting terms of ordrer higher than $\epsilon$. Note that (5.17) is relatively simple because it involves terms of order 1 and $\epsilon$ only: no $O\left(\epsilon^{2}\right)$ are required to preserve the Hamiltonian structure. Of course, if the equation were to be rewritten in terms of $\zeta$, using (5.5) as an exact transformation, then the corresponding bracket and Hamiltonian would include extra $O\left(\epsilon^{2}\right)$ terms. An advantage of a near-identity transformation results from the fact that (5.12) and (5.17) have the same linear part; therefore the sum of results that have been derived for the linearized vorticity equations can be exploited directly in terms of $\eta$.

\subsection{Vlasov-Poisson equation}

The one-dimensional Vlasov-Poisson equation is also generated by the bracket (5.1). In standard notation, $y=v$ is the particle velocity in the $x$ direction, and $\zeta=f(x, v)$ is the distribution function. For clarity, we will use the standard notation, and we rewrite

$$
\{\mathcal{F}, \mathcal{G}\}=\iint f\left[\frac{\delta \mathcal{F}}{\delta f}, \frac{\delta \mathcal{G}}{\delta f}\right] \mathrm{d} x \mathrm{~d} v
$$

with

$$
[f, g]:=\frac{1}{m}\left(\frac{\partial f}{\partial x} \frac{\partial g}{\partial v}-\frac{\partial f}{\partial v} \frac{\partial g}{\partial x}\right)
$$

The Hamiltonian is given by

$$
\mathcal{H}=\frac{1}{2} \iint m v^{2} f \mathrm{~d} x \mathrm{~d} v+\frac{1}{8 \pi} \int E^{2} \mathrm{~d} x,
$$

where the electric field $E(x)$ is related to the distribution function through the Poisson equation

$$
\frac{\partial E}{\partial x}=-\frac{\partial^{2} \Phi}{\partial x^{2}}=4 \pi e\left(\int f \mathrm{~d} v-N\right),
$$


$\Phi$ being the potential, $N$ a fixed background density, and $m$ and $e$ the particle mass and charge, respectively.

From (5.18)-(5.19), one can derive the Vlasov equation

$$
f_{t}+v f_{x}+\frac{e}{m} E f_{v}=0 .
$$

Considering the decomposition (4.3) in a basic distribution $f_{0}(v)$ and an $O(\epsilon)$ disturbance, we write the disturbance evolution equation

$$
f_{t}+v f_{x}+\frac{e}{m} f_{0}^{\prime} E+\epsilon \frac{e}{m} E f_{v}=0 .
$$

The free energy can be constructed similarly to that for the vorticity equation, with $\psi_{0}(\cdot)$ in $(5.13)$ replaced by $\frac{1}{2} m v^{2}+e \Phi_{0}$ which is functionally dependent on $f_{0}$. It is then found that

$$
\begin{aligned}
\mathcal{F}_{f} & =\frac{1}{8 \pi} \int E^{2} \mathrm{~d} x-\iint\left[\frac{1}{2} \frac{\partial}{\partial f_{0}}\left(\frac{m v^{2}}{2}\right) f^{2}+\frac{\epsilon}{6} \frac{\partial^{2}}{\partial f_{0}^{2}}\left(\frac{m v^{2}}{2}\right) f^{3}\right] \mathrm{d} x \mathrm{~d} v+O\left(\epsilon^{2}\right) \\
& =\frac{1}{8 \pi} \int E^{2} \mathrm{~d} x-m \iint\left[\frac{v}{2 f_{0}^{\prime}} f^{2}+\frac{\epsilon}{6 f_{0}^{\prime 2}}\left(1-\frac{v f_{0}^{\prime \prime}}{f_{0}^{\prime}}\right) f^{3}\right] \mathrm{d} x \mathrm{~d} v+O\left(\epsilon^{2}\right) .
\end{aligned}
$$

We now introduce the transformation (5.4)-(5.5) which can be rewritten as

$$
\eta=f-\epsilon \frac{\partial}{\partial v}\left(\frac{f^{2}}{2 f_{0}^{\prime}}\right) \text { and } f=\eta+\epsilon \frac{\partial}{\partial v}\left(\frac{\eta^{2}}{2 f_{0}^{\prime}}\right)+O\left(\epsilon^{2}\right) .
$$

It is such that the dynamics of $\eta$ is generated up to $O(\epsilon)$ by the Poisson operator

$$
\mathrm{J}_{0}=\frac{f_{0}^{\prime}}{m} \frac{\partial}{\partial x} .
$$

Introducing (5.22) in (5.21), we can compute the Hamiltonian for $\eta$. Compared to the vortex dynamics case, a simplification occurs because the Poisson equation is unchanged by the transformation:

$$
\frac{\partial E}{\partial x}=-\frac{\partial^{2} \Phi}{\partial x^{2}}=4 \pi e\left(\int f \mathrm{~d} v\right)=4 \pi e\left(\int \eta \mathrm{d} v\right),
$$

so that the first term in the right-hand side of (5.21) does not lead to a $O(\epsilon)$ contribution. The other two terms are readily simplified using integration by parts, and finally yield the truncated Hamiltonian

$$
\mathcal{F}_{\eta}=\frac{1}{8 \pi} \int E^{2} \mathrm{~d} x-\frac{m}{2} \iint\left(\frac{v}{f_{0}^{\prime}} \eta^{2}-\frac{\epsilon}{3 f_{0}^{\prime 2}} \eta^{3}\right) \mathrm{d} x \mathrm{~d} v .
$$

The evolution equation for $\eta$ is then readily obtained from (5.23) and (5.24). It is given by

$$
\eta_{t}=-v \eta_{x}-\frac{e}{m} f_{0}^{\prime} E+\frac{\epsilon}{f_{0}^{\prime}} \eta \eta_{x}
$$

Of course, this equation can also be found from (5.20) and (5.22).

\section{Stratified fluid and two-dimensional MHD}

The bracket for stratified fluid and two-dimensional MHD [29, 37], defined by

$$
\{\mathcal{F}, \mathcal{G}\}=\iint\left(\zeta\left[\frac{\delta \mathcal{F}}{\delta \zeta}, \frac{\delta \mathcal{G}}{\delta \zeta}\right]+\rho\left[\frac{\delta \mathcal{F}}{\delta \rho}, \frac{\delta \mathcal{G}}{\delta \zeta}\right]+\rho\left[\frac{\delta \mathcal{F}}{\delta \zeta}, \frac{\delta \mathcal{G}}{\delta \rho}\right]\right) \mathrm{d} x \mathrm{~d} y
$$

corresponds to the Poisson operator

$$
\mathbf{J}=\left(\begin{array}{cc}
-[\zeta, \cdot] & -[\rho, \cdot] \\
-[\rho, \cdot] & 0
\end{array}\right)
$$


where $(\zeta, \rho)$ are the dyamical variables. As for the Euler bracket, we consider a basic solution that depends only on $y, \zeta_{0}(y)$ and $\rho_{0}(y)$, and we introduce a decomposition of the form (4.3). From now on $(\zeta, \rho)$ thus designates the $O(\epsilon)$ disturbance to the basic solution. The leading-order Poisson operator which generates the linear evolution for $(\zeta, \rho)$ is given by

$$
\mathrm{J}_{0}=\left(\begin{array}{cc}
\zeta_{0}^{\prime} & \rho_{0}^{\prime} \\
\rho_{0}^{\prime} & 0
\end{array}\right) \frac{\partial}{\partial x}
$$

We are seeking new variables $(\eta, \sigma)$ such that condition (4.5) is satisfied. Rather than using a general near-identity transformation, we can exploit the close analogy between the bracket (6.1) and the Euler bracket, and use the procedure based on dynamical accessibility. From (6.2), it is clear that the new variables will have a constant average in the $x$ direction and we can expect the new variables to be given by the old ones minus their average part (up to a $\partial_{x}$ term which can be shown to be irrelevant), as observed in Section 5. To obtain this average, we consider the dynamical accessibility condition

$$
\begin{aligned}
\epsilon\left(\begin{array}{c}
\zeta \\
\rho
\end{array}\right) & =(\exp \{-\mathcal{G}, \cdot\}-\text { Id })\left(\begin{array}{c}
\zeta_{0} \\
\rho_{0}
\end{array}\right) \\
& =\epsilon\left(\begin{array}{c}
g_{x} \zeta_{0}^{\prime}+h_{x} \rho_{0}^{\prime} \\
g_{x} \rho_{0}^{\prime}
\end{array}\right)+\frac{\epsilon^{2}}{2}\left(\begin{array}{c}
{\left[g, g_{x} \zeta_{0}^{\prime}\right]+\left[h, g_{x} \rho_{0}^{\prime}\right]+\left[g, h_{x} \rho_{0}^{\prime}\right]} \\
{\left[g, g_{x} \rho_{0}^{\prime}\right]}
\end{array}\right)+O\left(\epsilon^{3}\right),
\end{aligned}
$$

where $g$ and $h$ are arbitrary functions and

$$
\mathcal{G}=\iint(g \zeta+h \rho) \mathrm{d} x \mathrm{~d} y .
$$

Taking the $x$-average of (6.3), one finds with some effort

$$
\left(\begin{array}{c}
\bar{\zeta} \\
\bar{\rho}
\end{array}\right)=\frac{\epsilon}{2} \frac{\partial}{\partial y}\left(\begin{array}{c}
\zeta_{0}^{\prime} \overline{g_{x}^{2}}+2 \rho_{0}^{\prime} \overline{g_{x} h_{x}} \\
\rho_{0}^{\prime} \overline{g_{x}^{2}}
\end{array}\right)+O\left(\epsilon^{2}\right) .
$$

Eq. (6.3) also yields the relationship between $(g, h)$ and $(\zeta, \rho)$, namely

$$
\left(\begin{array}{l}
g_{x} \\
h_{x}
\end{array}\right)=\frac{1}{\rho_{0}^{\prime}}\left(\begin{array}{c}
\rho \\
\zeta-\zeta_{0}^{\prime} \rho / \rho_{0}^{\prime}
\end{array}\right)+O(\epsilon)
$$

so that the average fields can be written

$$
\left(\begin{array}{c}
\bar{\zeta} \\
\bar{\rho}
\end{array}\right)=\epsilon \frac{\partial}{\partial y}\left[\frac{1}{2 \rho_{0}^{\prime}}\left(2 \overline{\rho \zeta}-\frac{\zeta_{0}^{\prime}}{\rho^{2}} / \rho_{0}^{\prime}\right)\right]+O\left(\epsilon^{2}\right) .
$$

This suggests the transformation from $(\zeta, \rho)$ to the new variables $(\eta, \sigma)$ defined by

$$
\left(\begin{array}{c}
\eta \\
\sigma
\end{array}\right)=\left(\begin{array}{c}
\zeta \\
\rho
\end{array}\right)-\epsilon \frac{\partial}{\partial y}\left[\frac{1}{2 \rho_{0}^{\prime}}\left(\begin{array}{c}
2 \rho \zeta-\zeta_{0}^{\prime} \rho^{2} / \rho_{0}^{\prime} \\
\rho^{2}
\end{array}\right)\right] .
$$

It can be verified that the bracket for those variables is given by $\mathrm{J}_{0}+O\left(\epsilon^{2}\right)$, with $\mathrm{J}_{0}$ defined by $(6.2)$, as required. The inverse transformation is readily obtained as

$$
\left(\begin{array}{c}
\zeta \\
\rho
\end{array}\right)=\left(\begin{array}{l}
\eta \\
\sigma
\end{array}\right)+\epsilon \frac{\partial}{\partial y}\left[\frac{1}{2 \rho_{0}^{\prime}}\left(\begin{array}{c}
2 \sigma \eta-\zeta_{0}^{\prime} \sigma^{2} / \rho_{0}^{\prime} \\
\sigma^{2}
\end{array}\right)\right]+O\left(\epsilon^{2}\right) .
$$

With these results, we can derive the Hamiltonian equations describing the weakly nonlinear evolution of a disturbance in a stratified fluid and in two-dimensional MHD.

\subsection{Stratified fluid}

The equations for an incompressible stratified fluid are derived from the bracket (6.1) and the Hamiltonian [36, 37]

$$
\mathcal{H}=\iint\left(\frac{1}{2} \rho|\nabla \psi|^{2}+\rho g y\right) \mathrm{d} x \mathrm{~d} y .
$$


The dynamical variables $\zeta$ and $\rho$ represent the (mass-weighted) vorticity and density, and the coordinate $y$ is vertical. The streamfunction $\psi$ is related to the vorticity through

$$
\zeta=\nabla \cdot(\rho \nabla \psi)
$$

The evolution equations take the form

$$
\begin{aligned}
\zeta_{t}+[\psi, \zeta]+\left[\rho, g y-|\nabla \psi|^{2} / 2\right] & =0 \\
\rho_{t}+[\psi, \rho] & =0 .
\end{aligned}
$$

We now consider a basic solution consisting of the parallel flow $U(y)$ and stratification $\rho_{0}(y)$, so that the basic vorticity is given by

$$
\zeta_{0}=-\left(\rho_{0} U\right)^{\prime} .
$$

To $O(\epsilon)$, a disturbance to this basic solution obeys the evolution equations

$$
\begin{aligned}
\zeta_{t}+U \zeta_{x}+\zeta_{0}^{\prime} \psi_{x}+\left(g-U U^{\prime}\right) \rho_{x}-\rho_{0}^{\prime} U \psi_{x y} & \\
+\epsilon\left([\psi, \zeta]+\rho_{0}^{\prime} \nabla \psi \cdot \nabla \psi_{x}+\left[\rho, U \psi_{y}\right]\right) & =0 \\
\rho_{t}+U \rho_{x}+\rho_{0}^{\prime} \psi_{x}+\epsilon[\psi, \rho] & =0
\end{aligned}
$$

with the streamfuction/vorticity relation given by

$$
\zeta=\nabla \cdot\left(\rho_{0} \nabla \psi\right)-(U \rho)_{y}+\epsilon \nabla \cdot(\rho \nabla \psi) .
$$

The free energy can now be computed using the Casimir functionals. Computations detailed in Appendix C give

$$
\begin{aligned}
\mathcal{F}_{(\eta, \sigma)}= & \iint\left\{\frac{1}{2} \rho_{0}|\nabla \varphi|^{2}-\frac{U}{\rho_{0}^{\prime}} \eta \sigma+\frac{1}{2 \rho_{0}^{\prime}}\left(U U^{\prime}+\frac{\zeta_{0}^{\prime} U}{\rho_{0}^{\prime}}-g\right) \sigma^{2}\right. \\
& \left.+\epsilon\left[\frac{1}{2} \sigma|\nabla \varphi|^{2}+\rho_{0} \nabla \varphi \cdot \nabla \vartheta+\frac{U^{\prime}}{2 \rho_{0}^{\prime 2}} \eta \sigma^{2}-\frac{1}{6 \rho_{0}^{\prime 3}}\left[2 \zeta_{0}^{\prime} U^{\prime}-\rho_{0}^{\prime}\left(U U^{\prime}\right)^{\prime}\right] \sigma^{3}\right]\right\} \mathrm{d} x \mathrm{~d} y .
\end{aligned}
$$

Here, $\varphi$ and $\vartheta$ are defined by the decomposition $\psi=\varphi+\epsilon \vartheta$ of the streamfunction. From (6.10) and (6.4), we find these to be given in terms of the new dependent variables $(\eta, \sigma)$ by

$$
\begin{aligned}
\nabla \cdot\left(\rho_{0} \nabla \varphi\right) & =\eta+\frac{\partial}{\partial y}(U \sigma) \\
\nabla \cdot\left(\rho_{0} \nabla \vartheta\right) & =\frac{\partial}{\partial y}\left(\frac{\eta \sigma}{\rho_{0}^{\prime}}-\frac{\zeta_{0}^{\prime} \sigma^{2}}{2 \rho_{0}^{\prime}}\right)+\frac{\partial}{\partial y}\left[U \frac{\partial}{\partial y}\left(\frac{\sigma^{2}}{2 \rho_{0}^{\prime}}\right)\right]-\nabla \cdot(\sigma \nabla \varphi) .
\end{aligned}
$$

The equations of motion in terms of $(\eta, \sigma)$ are derived by applying the Poisson operator $\mathrm{J}_{0}$ given by (6.2) to the functional dervatives of $\mathcal{F}_{(\eta, \sigma)}$, computed in Appendix C. The final result reads

$$
\begin{aligned}
\eta_{t}= & -U \eta_{x}-\zeta_{0}^{\prime} \varphi_{x}-\left(g-U U^{\prime}\right) \sigma_{x}+\rho_{0}^{\prime} U \varphi_{x y} \\
& +\epsilon\left\{-\zeta_{0}^{\prime} \vartheta_{x}-\rho_{0}^{\prime} U \vartheta_{x y}-\rho_{0}^{\prime} \nabla \varphi \cdot \nabla \varphi_{x}+\left(\varphi_{y} \eta\right)_{x}\right. \\
& \left.-\left[\left(U \varphi_{y}\right)_{y} \sigma\right]_{x}+\frac{U^{\prime}}{\rho_{0}^{\prime}}(\eta \sigma)_{x}-\frac{1}{\rho_{0}^{\prime 2}}\left[\zeta_{0}^{\prime} U^{\prime}+\rho_{0}^{\prime}\left(U U^{\prime}\right)^{\prime}\right] \sigma \sigma_{x}\right\} \\
\sigma_{t}= & -U \sigma_{x}-\rho_{0}^{\prime} \varphi_{x}+\epsilon\left[-\rho_{0}^{\prime} \vartheta_{x}+\left(\varphi_{y} \sigma\right)_{x}+\frac{U^{\prime}}{\rho_{0}^{\prime}} \sigma \sigma_{x}\right] .
\end{aligned}
$$

It can be checked that these equations are also found directly by mnaipulating (6.4), (6.5) and (6.9). 


\subsection{Two-dimensional MHD}

The equations for two-dimensional MHD with the magnetic field in the plane are Hamiltonian with the bracket (6.1), where $\zeta$ is to be interpreted as the vorticity and $\rho$ as the magnetic potential (often denoted by $A)$. The corresponding Hamiltonian $[29,30]$ is

$$
\mathcal{H}=\frac{1}{2} \iint\left(|\nabla \psi|^{2}+|\nabla \rho|^{2}\right) \mathrm{d} x \mathrm{~d} y,
$$

where $\psi$ is the streamfunction which, according to our previous sign convention, is defined by $\nabla^{2} \psi=\zeta$. The evolution equations are then of the form

$$
\begin{aligned}
\zeta_{t}+[\psi, \zeta]+\left[\rho, \nabla^{2} \rho\right] & =0 \\
\rho_{t}+[\psi, \rho] & =0 .
\end{aligned}
$$

The basic solution we consider is again a parallel flow $U(y)$, with the magnetic potential $\rho_{0}(y)$, corresponding to a magnetic field parallel to the streamlines. The evolution of a small disturbance to this basic solution is governed by

$$
\begin{aligned}
\zeta_{t}+U \zeta_{x}+\zeta_{0}^{\prime} \psi_{x}-\rho_{0}{ }^{\prime \prime \prime} \rho_{x}+\rho_{0}^{\prime} \nabla^{2} \rho_{x}+\epsilon\left\{[\psi, \zeta]+\left[\nabla^{2} \rho, \rho\right]\right\} & =0 \\
\rho_{t}+U \rho_{x}+\rho_{0}^{\prime} \psi_{x}+\epsilon[\psi, \rho] & =0
\end{aligned}
$$

where each prime denotes a derivative with respect to $y$.

The derivation of the free energy for this system closely follows that for the stratified fluid: decomposing $\psi=\varphi+\epsilon \vartheta$, with

$$
\nabla^{2} \varphi=\eta, \quad \nabla^{2} \vartheta=\frac{\partial}{\partial y}\left(\frac{\eta \sigma}{\rho_{0}^{\prime}}-\frac{\zeta_{0}^{\prime} \sigma^{2}}{2 \rho_{0}^{\prime 2}}\right),
$$

computations detailed in Appendix D lead to

$$
\begin{aligned}
\mathcal{F}_{(\eta, \sigma)}= & \iint\left\{\frac{1}{2}\left(|\nabla \phi|^{2}+|\nabla \sigma|^{2}\right)-\frac{U}{\rho_{0}^{\prime}} \eta \sigma+\frac{1}{2 \rho_{0}^{\prime}}\left(\frac{\zeta_{0}^{\prime} U}{\rho_{0}^{\prime}}+\rho_{0}^{\prime \prime \prime}\right) \sigma^{2}\right. \\
& \left.+\epsilon\left[\nabla \varphi \cdot \nabla \vartheta+\frac{U^{\prime}}{2 \rho_{0}^{\prime 2}} \eta \sigma^{2}+\frac{1}{2 \rho_{0}^{\prime}} \sigma^{2} \nabla^{2} \sigma_{y}-\frac{1}{6 \rho_{0}^{\prime 3}}\left(2 \zeta_{0}^{\prime} U^{\prime}+\rho_{0}^{\prime} \rho_{0}^{\prime \prime \prime \prime}\right) \sigma^{3}\right]\right\} \mathrm{d} x \mathrm{~d} y .
\end{aligned}
$$

Applying the Poisson operator (6.2), one can finally find the evolution equations

$$
\begin{aligned}
\eta_{t}= & -U \eta_{x}-\zeta_{0}^{\prime} \varphi_{x}-\rho_{0}^{\prime} \nabla^{2} \sigma_{x}+\rho_{0}^{\prime \prime \prime \prime} \sigma_{x} \\
& +\epsilon\left[-\zeta_{0}^{\prime} \vartheta_{x}+\left(\varphi_{y} \eta\right)_{x}+\frac{U^{\prime}}{\rho_{0}^{\prime}}(\eta \sigma)_{x}-\frac{1}{\rho_{0}^{\prime 2}}\left(\zeta_{0}^{\prime} U^{\prime}+\rho_{0}^{\prime} \rho_{0}^{\prime \prime \prime \prime}\right) \sigma \sigma_{x}\right. \\
& \left.+\left(\sigma \nabla^{2} \sigma_{y}\right)_{x}-\rho_{0}^{\prime} \nabla^{2} \frac{2}{x \partial y}\left(\frac{\sigma^{2}}{2 \rho_{0}^{\prime}}\right)\right] \\
\sigma_{t}= & -U \sigma_{x}-\rho_{0}^{\prime} \varphi_{x}+\epsilon\left[-\rho_{0}^{\prime} \vartheta_{x}+\left(\varphi_{y} \sigma\right)_{x}+\frac{U^{\prime}}{\rho_{0}^{\prime}} \sigma \sigma_{x}\right] .
\end{aligned}
$$

Again, these equations can be verified by using directly (6.4), (6.5) and (6.15).

\section{Discussion}

We have described beatification, a general method for obtaining weakly nonlinear Hamiltonian dynamical systems from a variety of finite and infinite-dimensional noncanonical Hamiltonian systems. We have seen that unlike canonical systems, for which the Poisson matrix is constant, perturbation theory for noncanoncial systems possesses the additional necessity of simultaneously expanding the Poisson bracket and the Hamiltonian. This could have been done for any known orbit or point in phase space, but here we expanded about a given equilibrium solution and obtained weakly nonlinear 


\begin{tabular}{lcccc}
\hline & Vortex dynamics & Vlasov-Poisson & Stratified fluid & 2-D MHD \\
\hline \hline Bracket & $(5.2)$ & $(5.23)$ & $(6.2)$ & $(6.2)$ \\
Free energy & $(5.16)$ & $(5.24)$ & $(6.11)$ & $(6.16)$ \\
Equations & $(5.17)$ & $(5.25)$ & $(6.13)$ & $(6.17)$ \\
\hline
\end{tabular}

Table 1: Equation numbers for the Hamiltonian structures and evolution equations in terms of the transformed variables.

systems for our examples. Table 7 gives the locations of the results for the four infinite-dimensional systems we considered.

Beatification can also be applied to the large class of Hamiltonian plasma reduced fluid models that have Poisson bracket (Jacobian) nonlinearities. Such models have additional scalar fields that describe two-fluid or extended MHD effects, the effects of gyroviscosity, which gives rise to nondissipative momentum transport in magnetized plasma [38, 39, 40,41,42,43, 44, 45, 46], and electromagnetic effects [47, 48]. All these models fit within the theory of Ref. [25], and the general beatification transformation can be worked out for all of them. Although these models are twodimensional, they can be extended by geometric aspect ratio expansion to what are referred to as weakly three-dimensional models [49], where the third dimension enters the equations of motion linearly.

More general Hamiltonian field theories have more complicated Poisson brackets than those of Table 7 considered here. For example, the bracket for three-dimensional MHD [3] has Lie-Poisson form, but in full generality it has in addition to the velocity and magnetic fields the density and entropy, giving a total of eight variables. Although this complicates significantly the beatification transformation, calculations like those presented in the present work can be done for this system. In fact, even the rather complicated magnetofluid model of full extended MHD, which includes Hall drift as well as electron inertia effects, is amenable, since we now have the noncanonical Poisson bracket, one that is not of Lie-Poisson form, for this full system [50, 51]. This full system includes Hall MHD [52] and inertial MHD as Hamiltonian subsystems and, because of the transformations given in [51], we see that beatification of only the Hall bracket is sufficient for beatification of inertial MHD and, indeed, the entire extended MHD. Similarly, the complicated bracket for the Vlasov-Maxwell system $[53,4,54,55]$ can be beatified.

Another application of the beatification procedure is to Hamiltonian systems governed by brackets that emerge from Dirac's constraint theory. Such systems have Poisson brackets called Dirac brackets, an examples being the Poisson brackets for the incompressible fluid [56, 57, 58], incompressible MHD [57, 58], and the modified Hasegawa-Mima equation [59]. Brackets, even more general than the conventional Dirac bracket [60] can be beatified.

The beatification procedure proposed in this paper lays the groundwork for the canonization of weakly nonlinear Hamiltonian systems. Because the brackets $J_{0}$ in the new coordinates are exactly those governing the linearized dynamics, the further coordinate transformations required to canonize weakly nonlinear systems are exactly those that canonize linear systems. We demonstrate this by explicitly carrying out the full canonization of the Vlasov-Poisson system considered in Section 5.2 in terms of action-angle variables, setting the stage for the perturbation theory proposed in [61]. This is detailed in Appendix E. Details of this are contained in [62], where equations for interacting continuous spectra analogous to the three-wave problem in finite dimensions were obtained. Similar calculations can be performed for fluid shear flow dynamics that have similar continuous spectra $[33,63,64]$, and the relationship of this approach to previous echo calculations [65] and recent rigorous results [66] would be of interest to pursue.

The degree to which the weakly nonlinear systems obtained here accurately track the full dynamics will depend on a case-by-case basis on the system under consideration, but some generic comments can be made. Clearly one would expect a better approximation the closer one chooses an initial condition to the equilibrium state and the smaller the numerical coefficients of the nonlinear terms in the Hamiltonian. For stable equilibria with Hamiltonians that have a convex linear part, our systems should give accurate frequency shifts due to nonlinearity for near equilibrium dynamics. However, to capture the extent of a basin of stability, if finite amplitude instability exists, would require extending the beatification transformation to higher order. For expansion about stable equilibria with Hamiltonians that have a nonconvex linear part, i.e., systems with negative energy modes, 
the situation is more delicate, even for the case of finite dimensions [67, 12] where explosive growth can occur. Progress has been made in understanding the structural stability of infinite-dimensional systems [68], but nonlinear behavior with negative energy modes complicates matters. For unstable equilibria, accurate slowing of the growth rate may be possible, but to understand the extent to which a mode can grow would also require a higher order expansion.

The beatification transformations considered in this paper flattened the Poisson bracket to first order. In recent work this has been generalized by flattening to second order [69], which is necessary to obtain consistent dynamics if one expands about a state that is not an equilibrium state. Also, this higher order beatification can be used to address the accuracy problems mentioned above. Moreover, it has been shown [70] how to construct a beatification transformation in terms of an operator series that flattens the Poisson bracket to all orders. This and other examples will be recorded and explored in future work.

\section{Acknowledgments}

One of us (PJM) was supported by the US Department of Energy Contract No. DE-FG02-04ER54742. Both of us would like to acknowledge the hospitality of the Isaac Newton Institute for Mathematical Sciences, where the bulk of this work was performed during the 1996 program The Mathematics of Atmosphere and Ocean Dynamics.

\section{Appendices}

\section{A Two-spin system}

In this Appendix, we provide some details of the treatment of the two-spin system considered in Section 3.3.

\section{A.1 Canonical variables for the linearised system}

Consider the linearized equations for $z_{(\alpha)}$. They are generated by the Hamiltonian (3.10) with the bracket

$$
\{f, g\}_{0}=m B_{1} \sum_{\alpha=1}^{2} \varepsilon_{i j 1} \frac{\partial f}{\partial z_{(\alpha)}^{i}} \frac{\partial g}{\partial z_{(\alpha)}^{j}}
$$

obtained by linearising (3.8). One finds explicitly

$$
\left\{\begin{array}{l}
\dot{z}_{(\alpha)}^{1}=0 \\
\dot{z}_{(\alpha)}^{2}=m B_{1}\left(\lambda z_{(\alpha)}^{3}+a z_{(\alpha+1)}^{3}\right) \\
\dot{z}_{(\alpha)}^{3}=-m B_{1}\left(\lambda z_{(\alpha)}^{2}+a z_{(\alpha+1)}^{2}\right)
\end{array} \quad \alpha=1,2\right.
$$

The invariance of $z_{(\alpha)}^{1}$ follows from the invariance of the Casimir functions in the original system. We now seek action-angle variables for this system. Using $z:=\left(z_{(1)}^{2}, z_{(1)}^{3}, z_{(2)}^{2}, z_{(2)}^{3}\right)^{\mathrm{T}}$, we can rewrite the dynamics as

$$
\dot{z}=\mathrm{A} z
$$

where

$$
\mathrm{A}:=m B_{1}\left(\begin{array}{cccc}
0 & \lambda & 0 & a \\
-\lambda & 0 & -a & 0 \\
0 & a & 0 & \lambda \\
-a & 0 & -\lambda & 0
\end{array}\right)
$$

The vector $z$ can then be expanded in terms of the eigenvectors of $A$ :

$$
z=\Lambda_{(1)} x_{(1)}+\Lambda_{(2)} x_{(2)}+\text { c.c. }
$$

where

$$
x_{(1)}:=(-\mathrm{i},-1, \mathrm{i}, 1)^{\mathrm{T}} / 2 \quad \text { and } \quad x_{(2)}:=(\mathrm{i}, 1, \mathrm{i}, 1)^{\mathrm{T}} / 2
$$


correspond to the eigenvalues $\mathrm{i} \sigma_{(1)}:=\mathrm{i} B_{1}(1+2 a m)$ and $\mathrm{i} \sigma_{(2)}:=\mathrm{i} B_{1}$, respectively. In terms of the variables $\Lambda_{(\alpha)}$, the Poisson bracket (A.1) is

$$
\{f, g\}_{0}=-\mathrm{i} m B_{1} \sum_{\alpha=1}^{2}\left(\frac{\partial f}{\partial \Lambda_{(\alpha)}} \frac{\partial g}{\partial \Lambda_{(\alpha)}^{*}}-\frac{\partial f}{\partial \Lambda_{(\alpha)}^{*}} \frac{\partial g}{\partial \Lambda_{(\alpha)}}\right) .
$$

Action and angle variables $\left(J_{(\alpha)}, \theta_{(\alpha)}\right)$ are then defined by

$$
\Lambda_{(\alpha)}=\sqrt{\left|m B_{1}\right| J_{(\alpha)}} \exp \left(-\mathrm{i} s \theta_{(\alpha)}\right),
$$

where $s:=\operatorname{sign}\left(m B_{1}\right)$, so that the bracket becomes canonical:

$$
\{f, g\}_{0}=\sum_{\alpha=1}^{2}\left(\frac{\partial f}{\partial \theta_{(\alpha)}} \frac{\partial g}{\partial J_{(\alpha)}}-\frac{\partial f}{\partial J_{(\alpha)}} \frac{\partial g}{\partial \theta_{(\alpha)}}\right) .
$$

Correspondingly, the (quadratic) Hamiltonian (3.8) reduces to

$$
F=-s \sum_{\alpha=1}^{2} \sigma_{(\alpha)} J_{(\alpha)}+a z_{(1)}^{1} z_{(2)}^{1} .
$$

The last term is of course irrelevant, as the variables $z_{(1)}^{1}$ and $z_{(2)}^{1}$ do not appear in the bracket.

\section{A.2 Canonical perturbation theory for the weakly nonlinear system}

Using the mixed generating function $S\left(\theta_{(\alpha)}, I_{(\alpha)}\right)$, we transform $\left(J_{(\alpha)}, \theta_{(\alpha)}\right)$ into the new variables $\left(I_{(\alpha)}, \phi_{(\alpha)}\right)$, with

$$
\frac{\partial S}{\partial \theta_{(\alpha)}}=J_{(\alpha)} \quad \text { and } \quad \frac{\partial S}{\partial I_{(\alpha)}}=\phi_{(\alpha)}
$$

such that the new Hamiltonian is independent of $\phi_{(\alpha)}$. It is easy to check that this is achieved with

$$
S=\sum_{\alpha=1}^{2} \theta_{(\alpha)} I_{(\alpha)}-\frac{\epsilon}{2 m B_{1}}\left(c_{1}-c_{2}\right) \sqrt{I_{(1)} I_{(2)}} \sin \left(\theta_{(1)}-\theta_{(2)}\right) .
$$

The new Hamiltonian is obtained by averaging; it is given by

$$
F\left(I_{(\alpha)}\right)=-s \sum_{\alpha=1}^{2} \sigma_{(\alpha)} I_{(\alpha)}+a c_{1} c_{2}-\epsilon s a\left(c_{1}+c_{2}\right) I_{(1)} .
$$

The frequencies of the nonlinear system are thus given by $\sigma_{(1)}+\epsilon a\left(c_{1}+c_{2}\right)$ and $\sigma_{(2)}$. The exact relationship between $\left(I_{(\alpha)}, \phi_{(\alpha)}\right)$ and $\left(J_{(\alpha)}, \theta_{(\alpha)}\right)$ can be found from (A.4)-(A.5).

It is interesting to note that, due to the extreme simplicity of the system, one can integrate the weakly nonlinear equations without resorting to the canonical perturbation techniques. From (3.15), it is clear that the nonlinear coupling vanishes to the order of accuracy considered here if $\eta_{(\alpha)}^{1}=O(\epsilon)$ initially. Actually, this can be obtained for any initial condition by suitably redefining the decomposition between equilibrium solution and the disturbance. This decomposition is indeed partly arbitrary, and one can write the initial condition as

$$
\omega_{(\alpha)}^{1}(0)=m B_{1}+\epsilon z_{(\alpha)}^{1}(0)=m_{(\alpha)}^{\prime} B_{1},
$$

with $m_{(\alpha)}^{\prime}:=1+\epsilon z_{(\alpha)}^{1}(0) /\left(m B_{1}\right)$. With the second decomposition the disturbance has initially vanishing components in the direction of the external magnetic field, and $\eta_{(\alpha)}^{1}=O(\epsilon)$ for all time. The cubic terms in the Hamiltonian vanish, so that there is no weakly nonlinear effect at the order considered. Studying the linear system as above but with $m_{(1)}^{\prime} \neq m_{(2)}^{\prime}$, one finds the two frequencies $\sigma_{(1)}+\epsilon a\left(c_{1}+c_{2}\right)$ and $\sigma_{(2)}$ as obtained by the perturbation theory. 


\section{B Extension of the Zakharov-Piterbarg transformation}

The variables $x, y, \zeta(x, y)$ are transformed into $x, z, \eta_{\mathrm{C}}(x, z)$ defined by

$$
z[x, y, \zeta]=y_{0}\left[\zeta_{0}(y)+\epsilon \zeta(x, y)\right], \quad \eta_{\mathrm{C}}[x, y, \zeta]=\left.\epsilon^{-1} \frac{\mathrm{d} \zeta_{0}}{\mathrm{~d} y_{0}}\right|_{z}(z-y) .
$$

These relations can be inverted according to

$$
y\left[x, z, \eta_{\mathrm{C}}\right]=z-\left.\epsilon \frac{\mathrm{d} y_{0}}{\mathrm{~d} \zeta_{0}}\right|_{\zeta_{0}(z)} \eta_{\mathrm{C}}(x, z), \quad \zeta\left[x, z, \eta_{\mathrm{C}}\right]=\epsilon^{-1}\left[\zeta_{0}(z)-\zeta_{0}(y)\right]
$$

Roughly speaking, one can say that the transformation exchanges the roles of the independent and dependent variables: $z$ is essentially defined in terms of the vorticity, while $\eta_{c}$ is defined in terms of parcel displacement - the expressions are somewhat complicated by the fact that the transformation is near-identity so that $z$ is a distance and $\eta_{\mathrm{C}}$ a disturbance vorticity. Noting that

$$
\mathrm{d} y=\left[1-\epsilon \frac{\partial}{\partial z}\left(\left.\frac{\mathrm{d} y_{0}}{\mathrm{~d} \zeta_{0}}\right|_{\zeta_{0}(z)} \eta_{\mathrm{C}}\right)\right] \mathrm{d} z
$$

we find a condition analogous to (5.6) for the well-definiteness of the transformation. We now compute the bracket (5.1) in terms of $x, z, \eta_{\mathrm{C}}$. From (B.1)-(B.2), one can see that $\zeta(x, y)$ and $\eta_{\mathrm{C}}(x, z)$ are functionally related through

$$
\zeta(x, y)=\epsilon^{-1} \int\left\{\zeta_{0}(z)-\zeta_{0}\left[z-\left.\epsilon \frac{\mathrm{d} y_{0}}{\mathrm{~d} \zeta_{0}}\right|_{\zeta_{0}(z)} \eta_{\mathrm{C}}(x, z)\right]\right\} \delta\left(z-y_{0}\left[\zeta_{0}(y)+\epsilon \zeta(x, y)\right]\right) \mathrm{d} z
$$

Taking the variation of this equality leads to

$$
\begin{aligned}
\delta \zeta(x, y) & =\left.\left.\int \frac{\mathrm{d} \zeta_{0}}{\mathrm{~d} y_{0}}\right|_{y} \frac{\mathrm{d} y_{0}}{\mathrm{~d} \zeta_{0}}\right|_{\zeta_{0}(z)} \delta \eta(x, z) \delta\left(z-y_{0}\left[\zeta_{0}(y)+\epsilon \zeta(x, y)\right]\right) \mathrm{d} z \\
& +\left.\left.\frac{\partial}{\partial z}\left\{\zeta_{0}(z)-\zeta_{0}\left[z-\left.\epsilon \frac{\mathrm{d} y_{0}}{\mathrm{~d} \zeta_{0}}\right|_{\zeta_{0}(z)} \eta_{\mathrm{C}}(x, z)\right]\right\}\right|_{z=y_{0}\left[\zeta_{0}(y)+\epsilon \zeta(x, y)\right]} \frac{\mathrm{d} y_{0}}{\mathrm{~d} \zeta_{0}}\right|_{\zeta_{0}(y)+\epsilon \zeta(x, y)} \delta \zeta(x, y)
\end{aligned}
$$

which can be rewritten as

$$
\begin{aligned}
& \left.\left.\frac{\partial \zeta_{0}}{\partial y_{0}}\right|_{y} \frac{\mathrm{d} y_{0}}{\mathrm{~d} \zeta_{0}}\right|_{\zeta_{0}(y)+\epsilon \zeta(x, y)}\left[1-\epsilon \frac{\partial}{\partial z}\left(\left.\frac{\mathrm{d} y_{0}}{\mathrm{~d} \zeta_{0}}\right|_{\zeta_{0}(z)} \eta_{\mathrm{C}}(x, z)\right)\right]_{z=y_{0}\left[\zeta_{0}(y)+\epsilon \zeta(x, y)\right]} \delta \zeta(x, y) \\
= & \left.\left.\int \frac{\mathrm{d} \zeta_{0}}{\mathrm{~d} y_{0}}\right|_{y} \frac{\mathrm{d} y_{0}}{\mathrm{~d} \zeta_{0}}\right|_{\zeta_{0}(z)} \delta \eta(x, z) \delta\left(z-y_{0}\left[\zeta_{0}(y)+\epsilon \zeta(x, y)\right]\right) \mathrm{d} z
\end{aligned}
$$

and finally as

$$
\delta \zeta(x, y)=\int\left[1-\epsilon \frac{\partial}{\partial z}\left(\left.\frac{\mathrm{d} y_{0}}{\mathrm{~d} \zeta_{0}}\right|_{\zeta_{0}(z)} \eta_{\mathrm{C}}(x, z)\right)\right]^{-1} \delta \eta(x, z) \delta\left(z-y_{0}\left[\zeta_{0}(y)+\epsilon \zeta(x, y)\right]\right) \mathrm{d} z
$$

Consider now the variation of an arbitrary functional $\mathcal{F}$, namely

$$
\delta \mathcal{F}=\iint \frac{\delta \mathcal{F}}{\delta \zeta(x, y)} \delta \zeta(x, y) \mathrm{d} x \mathrm{~d} y=\iint \frac{\delta \mathcal{F}}{\delta \eta_{\mathrm{C}}(x, z)} \delta \eta_{\mathrm{C}}(x, z) \mathrm{d} x \mathrm{~d} z
$$

Introducing (B.4), the first equality becomes

$$
\delta \mathcal{F}=\iiint\left[1-\epsilon \frac{\partial}{\partial z}\left(\left.\frac{\mathrm{d} y_{0}}{\mathrm{~d} \zeta_{0}}\right|_{\zeta_{0}(z)} \eta_{\mathrm{C}}(x, z)\right)\right]^{-1} \delta \eta(x, z) \delta\left(z-y_{0}\left[\zeta_{0}(y)+\epsilon \zeta(x, y)\right]\right) \frac{\delta \mathcal{F}}{\delta \zeta(x, y)} \mathrm{d} x \mathrm{~d} y \mathrm{~d} z
$$


From (B.3), it can be seen that the integration in $y$ can be carried out: defining $z^{\prime}$ by $z^{\prime}=y_{0}\left[\zeta_{0}(y)+\right.$ $\epsilon \zeta(x, y)]$, one finds

$$
\int \delta\left(z-y_{0}\left[\zeta_{0}(y)+\epsilon \zeta(x, y)\right]\right) \frac{\delta \mathcal{F}}{\delta \zeta(x, y)} \mathrm{d} y=\int\left[1-\epsilon \frac{\partial}{\partial z^{\prime}}\left(\left.\frac{\mathrm{d} y_{0}}{\mathrm{~d} \zeta_{0}}\right|_{\zeta_{0}\left(z^{\prime}\right)} \eta_{\mathrm{C}}\left(x, z^{\prime}\right)\right)\right] \delta\left(z-z^{\prime}\right) \frac{\delta \mathcal{F}}{\delta \zeta(x, y)} \mathrm{d} z^{\prime} .
$$

Substituting this result in the previous equation leads to

$$
\delta \mathcal{F}=\iint \frac{\delta \mathcal{F}}{\delta \zeta(x, y)} \delta \eta_{\mathrm{C}}(x, z) \mathrm{d} x \mathrm{~d} y,
$$

where $y$ is now related to $z, \eta_{\mathrm{C}}(x, z)$ through (B.2). Comparing with the second equality of (B.5) gives the result

$$
\frac{\delta \mathcal{F}}{\delta \zeta(x, y)}=\frac{\delta \mathcal{F}}{\delta \eta_{\mathrm{C}}(x, z)}
$$

analogous to that of Zakharov and Piterbarg. The bracket (5.1) can now be expressed in terms of the new variables:

$$
\begin{aligned}
\{\mathcal{F}, \mathcal{G}\} & =-\iint \frac{\delta \mathcal{F}}{\delta \zeta}\left[\zeta_{0}(y)+\epsilon \zeta(x, y), \frac{\delta \mathcal{G}}{\delta \zeta}\right] \mathrm{d} x \mathrm{~d} y \\
& =-\iint \frac{\delta \mathcal{F}}{\delta \eta_{\mathrm{C}}} \frac{\partial\left(\zeta_{0}(z), \frac{\delta \mathcal{G}}{\delta \eta_{\mathrm{C}}}\right)}{\partial(x, y)}\left|\frac{\partial(x, y)}{\partial(x, z)}\right| \mathrm{d} x \mathrm{~d} z \\
& =\left.\iint \frac{\mathrm{d} \zeta_{0}}{\mathrm{~d} y_{0}}\right|_{z} \frac{\delta \mathcal{F}}{\delta \eta_{\mathrm{C}}} \frac{\partial}{\partial x}\left(\frac{\delta \mathcal{G}}{\delta \eta_{\mathrm{C}}}\right) \mathrm{d} x \mathrm{~d} z .
\end{aligned}
$$

Correspondingly, the Poisson operator is given in terms of the variables $x, z, \eta_{\mathrm{C}}$ by $\mathrm{J}_{0}=\zeta_{0}^{\prime} \partial_{x}$.

It is easy to see that the transformation (B.1)-(B.2) is equivalent to the Zakharov-Piterbarg transformation when $\zeta_{0}\left(y_{0}\right)=\beta y_{0}$; indeed, one finds,

$$
z=y+\epsilon \beta^{-1} \zeta(x, y), \quad \eta_{\mathrm{C}}(x, z)=\zeta(x, y)
$$

which is equivalent to (5.10) and to their equation (6) (noting the correspondence $\left(\zeta, \eta_{\mathrm{C}}\right) \rightarrow(\Omega, \zeta)$ between our notation and theirs). That our transformation (5.4) is the leading order approximation to (B.1)-(B.2) can be verified by expanding the expression for $z$ in (B.1) to obtain

$$
z=y+\left.\epsilon \frac{\mathrm{d} y_{0}}{\mathrm{~d} \zeta_{0}}\right|_{\zeta_{0}(y)} \zeta(x, y)+\left.\frac{\epsilon^{2}}{2} \frac{\mathrm{d}^{2} y_{0}}{\mathrm{~d} \zeta_{0}^{2}}\right|_{\zeta_{0}(y)} \zeta^{2}(x, y)+O\left(\epsilon^{3}\right)
$$

Using the $O(\epsilon)$ approximation to eliminate the implicit $y$-dependence in terms of order $O(\epsilon)$, one can inverse this relation according to

$$
y=z-\left.\epsilon \frac{\mathrm{d} y_{0}}{\mathrm{~d} \zeta_{0}}\right|_{\zeta_{0}(z)} \zeta(x, z)+\left.\frac{\epsilon^{2}}{2} \frac{\mathrm{d}^{2} y_{0}}{\mathrm{~d} \zeta_{0}^{2}}\right|_{\zeta_{0}(z)} \zeta^{2}(x, z)+\frac{\epsilon^{2}}{2}\left(\left.\frac{\mathrm{d} y_{0}}{\mathrm{~d} \zeta_{0}}\right|_{\zeta_{0}(z)}\right)^{2} \frac{\partial}{\partial z}\left[\zeta^{2}(x, z)\right]+O\left(\epsilon^{3}\right) .
$$

Introducing this result into the definition (B.1) for $\eta_{\mathrm{C}}$ yields

$$
\eta_{\mathrm{C}}(x, z)=\zeta(x, z)-\frac{\epsilon}{2} \frac{\partial}{\partial z}\left[\left.\frac{\mathrm{d} y_{0}}{\mathrm{~d} \zeta_{0}}\right|_{\zeta_{0}(z)} \zeta^{2}(x, z)\right]+O\left(\epsilon^{2}\right),
$$

which is equivalent to $(5.4), z$ replacing $y$.

\section{Free energy for the stratified fluid}

The free energy for stratified fluids is obtained by combining (6.6) with the Casimir functionals for the bracket (6.1) which have the form

$$
\mathcal{C}_{1}=\iint C_{1}\left(\rho_{0}+\epsilon \rho\right) \mathrm{d} x \mathrm{~d} y, \quad \mathcal{C}_{2}=\iint\left(\zeta_{0}+\epsilon \zeta\right) C_{2}\left(\rho_{0}+\epsilon \rho\right) \mathrm{d} x \mathrm{~d} y,
$$


for arbitrary functions $C_{1}(\cdot)$ and $C_{2}(\cdot)$. The proper choice can be found to be

$$
C_{1}(\cdot)=-\int_{\rho_{0}}^{(\cdot)} h(\mu) \mathrm{d} \mu, \quad C_{2}(\cdot)=\psi_{0}(\cdot),
$$

where $\psi_{0}(\cdot)$ and $h:=\zeta_{0} \mathrm{~d} \psi_{0} / \mathrm{d} \rho_{0}+g y-U^{2} / 2$ are defined by their functional dependence on $\rho_{0}$. This dependence follows from the steady state condition for the basic solution. Adding these Casimir functionals to the Hamiltonian leads to the free energy in the form

$$
\begin{aligned}
\mathcal{F}_{(\zeta, \rho)}= & \iint\left\{\frac{1}{2}\left(\rho_{0}+\epsilon \rho\right)|\nabla \psi|^{2}+\frac{\mathrm{d} \psi_{0}}{\mathrm{~d} \rho_{0}} \zeta \rho-\epsilon^{-1} \int_{0}^{\rho}\left[h\left(\rho_{0}+\epsilon \mu\right)-h\left(\rho_{0}\right)\right] \mathrm{d} \mu\right. \\
& \left.+\epsilon^{-2}\left(\zeta_{0}+\epsilon \zeta\right)\left[\psi_{0}\left(\rho_{0}+\epsilon \rho\right)-\psi_{0}\left(\rho_{0}\right)-\epsilon \frac{\mathrm{d} \psi_{0}}{\mathrm{~d} \rho_{0}} \rho\right]\right\} \mathrm{d} x \mathrm{~d} y
\end{aligned}
$$

which can be expanded in $\epsilon$. Keeping only the $O(1)$ and $O(\epsilon)$ terms, we find

$$
\begin{aligned}
\mathcal{F}_{(\zeta, \rho)}= & \iint\left\{\frac{1}{2} \rho_{0}|\nabla \psi|^{2}+\frac{\mathrm{d} \psi_{0}}{\mathrm{~d} \rho_{0}} \zeta \rho+\frac{1}{2}\left(\zeta_{0} \frac{\mathrm{d}^{2} \psi_{0}}{\mathrm{~d} \rho_{0}^{2}}-\frac{\mathrm{d} h}{\mathrm{~d} \rho_{0}}\right) \rho^{2}\right. \\
& \left.+\epsilon\left[\frac{1}{2} \rho|\nabla \psi|^{2}+\frac{1}{6} \frac{\mathrm{d}^{2} \psi_{0}}{\mathrm{~d} \rho_{0}^{2}} \zeta \rho^{2}+\frac{1}{6}\left(\zeta_{0} \frac{\mathrm{d}^{3} \psi_{0}}{\mathrm{~d} \rho_{0}^{3}}-\frac{\mathrm{d}^{2} h}{\mathrm{~d} \rho_{0}^{2}}\right) \rho^{3}\right]\right\} \mathrm{d} x \mathrm{~d} y+O\left(\epsilon^{2}\right) .
\end{aligned}
$$

The next step is to introduce the variable transformation (6.4) into this free energy. We decompose the streamfunction according to $\psi=\varphi+\epsilon \vartheta$ and find $\varphi$ and $\vartheta$ given by (6.12). The terms in $|\nabla \psi|^{2}$ in (C.3) are then readily expressed as functions of $\varphi$ and $\vartheta$. Substitution of (6.4) into (C.3) yields terms in $\eta \sigma, \sigma^{2}, \eta \sigma^{2}$ and $\sigma^{3}$. Lengthy calculations, using extensively the relation

$$
\zeta_{0} \frac{\mathrm{d} \psi_{0}}{\mathrm{~d} \rho_{0}}-h=\frac{U^{2}}{2}-g y
$$

and its successive derivatives are necessary to put them in a simple form. This leads to the free energy (6.11).

The functional derivatives of $\mathcal{F}$ need to be calculated to derive the equations of motion. Using (6.12), one can find

$$
\begin{aligned}
\frac{\delta \mathcal{F}_{(\eta, \sigma)}}{\delta \eta}= & -\varphi-\frac{U^{\prime}}{\rho_{0}^{\prime}} \sigma+\epsilon\left(\frac{\varphi_{y} \sigma}{\rho_{0}^{\prime}}+\frac{U^{\prime}}{2 \rho_{0}^{\prime 2}} \sigma^{2}-\vartheta\right) \\
\frac{\delta \mathcal{F}_{(\eta, \sigma)}}{\delta \sigma}= & -\frac{U}{\rho_{0}^{\prime}} \eta+U \varphi_{y}+\frac{1}{\rho_{0}^{\prime}}\left(U U^{\prime}+\frac{\zeta_{0}^{\prime} U}{\rho_{0}^{\prime}}-g\right) \sigma \\
& +\epsilon\left\{\frac{1}{\rho_{0}^{\prime}} \varphi_{y} \eta-\frac{1}{2}|\nabla \varphi|^{2}-\frac{\zeta_{0}^{\prime}}{\rho_{0}^{\prime 2}} \varphi_{y} \sigma-\frac{1}{\rho_{0}^{\prime}}\left(U \varphi_{y}\right)_{y} \sigma\right. \\
& \left.+U \vartheta_{y}+\frac{U^{\prime}}{\rho_{0}^{\prime 2}} \eta \sigma-\frac{1}{2 \rho_{0}^{\prime 3}}\left[2 \zeta_{0}^{\prime} U^{\prime}+\rho_{0}^{\prime}\left(U U^{\prime}\right)^{\prime}\right] \sigma^{2}\right\}
\end{aligned}
$$

\section{Free energy for two-dimensional MHD}

We derive the free energy for two-dimensional MHD by adding to the Hamiltonian (6.14) a suitable Casimir functionals whose general form is again (C.1). The choice leading to a quadratic free energy is formally given by (C.2), with $h=\zeta_{0} \mathrm{~d} \psi_{0} / \mathrm{d} \rho_{0}-\nabla^{2} \rho_{0}$. The general form of the free energy is found to be

$$
\begin{aligned}
\mathcal{F}_{(\zeta, \rho)}= & \iint\left\{\frac{1}{2}\left(|\nabla \psi|^{2}+|\nabla \rho|^{2}\right)-\epsilon^{-1} \int_{0}^{\rho}\left[h\left(\rho_{0}+\epsilon \mu\right)-h\left(\rho_{0}\right)\right] \mathrm{d} \mu\right. \\
& \left.+\epsilon^{-2}\left(\zeta_{0}+\epsilon \zeta\right)\left[\psi_{0}\left(\rho_{0}+\epsilon \rho\right)-\psi_{0}\left(\rho_{0}\right)-\epsilon \frac{\mathrm{d} \psi_{0}}{\mathrm{~d} \rho_{0}} \rho\right]+\frac{\mathrm{d} \psi_{0}}{\mathrm{~d} \rho_{0}} \zeta \rho\right\} \mathrm{d} x \mathrm{~d} y
\end{aligned}
$$


and after expanding in $\epsilon$

$$
\begin{aligned}
\mathcal{F}_{(\zeta, \rho)}= & \iint\left\{\frac{1}{2}\left(|\nabla \psi|^{2}+|\nabla \rho|^{2}\right)+\frac{\mathrm{d} \psi_{0}}{\mathrm{~d} \rho_{0}} \zeta \rho+\frac{1}{2}\left(\zeta_{0} \frac{\mathrm{d}^{2} \psi_{0}}{\mathrm{~d} \rho_{0}^{2}}-\frac{\mathrm{d} h}{\mathrm{~d} \rho_{0}}\right) \rho^{2}\right. \\
& \left.+\epsilon\left[\frac{1}{2} \frac{\mathrm{d}^{2} \psi_{0}}{\mathrm{~d} \rho_{0}^{2}} \zeta \rho^{2}+\frac{1}{6}\left(\zeta_{0} \frac{\mathrm{d}^{3} \psi_{0}}{\mathrm{~d} \rho_{0}^{3}}-\frac{\mathrm{d}^{2} h}{\mathrm{~d} \rho_{0}^{2}}\right) \rho^{3}\right]\right\} \mathrm{d} x \mathrm{~d} y+O\left(\epsilon^{2}\right) .
\end{aligned}
$$

Introducing the variable change (6.5), with $\psi=\varphi+\epsilon \vartheta$, finally leads to the free energy in the form (6.16).

The variational derivatives of this free energy with respect to $\eta$ and $\sigma$ can then be evaluated; they are given by

$$
\begin{aligned}
\frac{\delta F_{(\eta, \sigma)}}{\delta \eta}= & -\varphi-\frac{U}{\rho_{0}^{\prime}} \sigma+\epsilon\left[-\vartheta+\frac{1}{\rho_{0}^{\prime}} \varphi_{y} \sigma+\frac{U^{\prime}}{2 \rho_{0}^{\prime 2}} \sigma^{2}\right] \\
\frac{\delta F_{(\eta, \sigma)}}{\delta \sigma}= & -\nabla^{2} \sigma-\frac{U}{\rho_{0}^{\prime}} \eta+\frac{1}{\rho_{0}^{\prime 2}}\left(\zeta_{0}^{\prime} U+\rho_{0}^{\prime} \rho^{(3)}\right) \sigma \\
& +\epsilon\left[\frac{1}{\rho_{0}^{\prime}} \varphi_{y} \eta-\frac{\zeta_{0}^{\prime}}{\rho_{0}^{\prime 2}} \phi_{y} \sigma+\frac{U^{\prime}}{\rho_{0}^{\prime}} \eta \sigma\right. \\
& \left.+\frac{1}{\rho_{0}^{\prime}} \sigma \nabla^{2} \sigma_{y}-\nabla^{2} \frac{\partial}{\partial y} \frac{\sigma^{2}}{2 \rho_{0}^{\prime}}-\frac{1}{2 \rho_{0}^{\prime 3}}\left(2 \zeta_{0}^{\prime} U^{\prime}+\rho_{0}^{\prime} \rho_{0}^{(4)}\right) \sigma^{2}\right] .
\end{aligned}
$$

\section{E Canonization for continuous spectra}

Once the weakly nonlinear equations are beatified, formulated as a Hamiltonian system with a constant Poisson operator $\mathrm{J}_{0}$, it is natural to seek the next step of canonization, i.e., transforming into a canonical form. Standard (canonical) perturbation methods, starting with the diagonalization of the leading-order Hamiltonian, can then be applied to study (and possibly integrate) the weakly nonlinear dynamics. Because the bracket $J_{0}$ found for the weakly nonlinear system is also that of the linearized system, both the canonization and the diagonalization of the leading-order Hamiltonian are achieved if one uses the action-angle variables of the linearized system. For finite-dimensional systems, those variables are easier to find (see Appendix A for an example). For infinite-dimensional systems, however, this can be a non-trivial task unless the equilibrium solution is simple. Indeed, the spectrum of the linear evolution operator generally contains a continuous part which needs to be treated carefully. Nevertheless, existing results provide action-angle variables corresponding to the continuous spectrum for the linearized vorticity and Vlasov-Poisson equations [71, 61, 33, 63, 64] that we have considered in Section 5. Here, we show how these results can be exploited to cast the weakly nonlinear equations in a very simple form, well-suited to start further investigations. We focus on the Vlasov-Poisson equation, as it is somewhat simpler than the vorticity equations. The latter could be treated equivalently with minor modifications.

The transformations rendering the linear Vlasov-Poisson equations into canonical form was first described in [71] and are intimately related to the Van Kampen generalized eigenmodes of the linearized system. We apply these transformations to the variable $\eta$. Schematically, the following successive changes of variables are made:

$$
\eta(x, v, t) \rightarrow \eta_{k}(v, t) \rightarrow E_{k}(u, t) \rightarrow J_{k}(u, t), \theta_{k}(u, t) .
$$

Here, $\eta_{k}$ is the Fourier transform of $\eta$ in $x, E_{k}$ are coordinates in which the linearized system is diagnonalized, and $\left(J_{k}, \theta_{k}\right)$ are the action-angle variables. The transformation from $\eta_{k}$ to $E_{k}$ can be seen as an integral transform defined by

$$
\eta_{k}(v, t)=\frac{\mathrm{i} k}{4 \pi e} \mathrm{G}\left[E_{k}(u, t)\right]:=\frac{\mathrm{i} k}{4 \pi e} \int_{-\infty}^{\infty} G_{k}(u, v) E_{k}(u, t) \mathrm{d} u,
$$

with the kernel

$$
G_{k}(u, v):=\varepsilon_{I}(k, v) \mathcal{P} \frac{1}{u-v}+\varepsilon_{R}(k, v) \delta(u-v),
$$


where $\varepsilon_{I}$ and $\varepsilon_{R}$ are the imaginary and real parts of the plasma dielectric function. The inverse transform can be shown to be

$$
E_{k}(u, t)=\frac{4 \pi e}{\mathrm{i} k} \tilde{\mathrm{G}}\left[\eta_{k}(v, t)\right]:=\frac{4 \pi e}{\mathrm{i} k} \int_{-\infty}^{\infty} \tilde{G}_{k}(u, v) \eta_{k}(v, t) \mathrm{d} v,
$$

with the kernel

$$
\tilde{G}_{k}(u, v):=\frac{1}{|\varepsilon(k, u)|^{2}}\left[\varepsilon_{I}(k, u) \mathcal{P} \frac{1}{u-v}+\varepsilon_{R}(k, u) \delta(u-v)\right] .
$$

The action/angle variables are then defined by

$$
E_{k}(u, t)=\sqrt{\frac{16\left|\varepsilon_{I}\right|}{|k| V|\varepsilon|^{2}} J_{k}(u, t)} \exp \left[-\mathrm{i} s_{k} \theta_{k}(u, t)\right],
$$

where $s_{k}:=\operatorname{sign} k$. In terms of these variables, the Poisson bracket (5.23) becomes canonical, i.e.

$$
\{\mathcal{F}, \mathcal{G}\}=\sum_{k=1}^{\infty} \int_{-\infty}^{\infty}\left(\frac{\delta \mathcal{F}}{\delta \theta_{k}} \frac{\delta \mathcal{G}}{\delta J_{k}}-\frac{\delta \mathcal{F}}{\delta J_{k}} \frac{\delta \mathcal{G}}{\delta \theta_{k}}\right) \mathrm{d} u
$$

and the quadratic part of the Hamiltonian is simply

$$
\mathcal{F}^{(2)}=\sum_{k=1}^{\infty} \int_{-\infty}^{\infty} \omega_{k}(u) J_{k}(u, t) \mathrm{d} u
$$

with the frequencies $\omega_{k}(u):=k u \operatorname{sign} \varepsilon_{I}(k, u)$.

To derive a canonical form of the Hamiltonian equations (5.25) describing the weakly nonlinear evolution of a disturbance [62], we only need to express the $O(\epsilon)$ (cubic) part of the Hamiltonian (5.24) in terms of the action-angle variables. Substituting (E.1) and integrating in $x$, one finds

$$
\begin{aligned}
& -\frac{m}{3} \iint \frac{\eta^{3}}{f_{0}^{\prime 2}} \mathrm{~d} x \mathrm{~d} v=\frac{\mathrm{i} m}{3(4 \pi e)^{3}} \sum_{k_{a}} \sum_{k_{b}} \int \frac{k_{a} k_{b} k_{c}}{f_{0}^{\prime 2}} \mathrm{~d} v \\
& \times \quad\left(\int_{-\infty}^{\infty} G_{k_{b}}\left(u_{a}, v\right) E_{k_{a}}\left(u_{a}, t\right) \mathrm{d} u_{a} \int_{-\infty}^{\infty} G_{k_{b}}\left(u_{b}, v\right) E_{k_{b}}\left(u_{b}, t\right) \mathrm{d} u_{b} \int_{-\infty}^{\infty} G_{k_{c}}\left(u_{c}, v\right) E_{k_{c}}\left(u_{c}, t\right) \mathrm{d} u_{c}\right),
\end{aligned}
$$

with $k_{a}+k_{b}+k_{c}=0$. This expression can be written in the standard form

$$
\mathcal{F}^{(3)}=\sum_{k_{a}} \sum_{k_{b}} \iiint I\left(k_{a}, k_{b}, k_{c}, u_{a}, u_{b}, u_{c}\right) E_{k_{a}}\left(u_{a}, t\right) E_{k_{b}}\left(u_{b}, t\right) E_{k_{a}}\left(u_{c}, t\right) \mathrm{d} u_{a} \mathrm{~d} u_{b} \mathrm{~d} u_{c},
$$

where $I(\cdots)$ is an interaction coefficient. To derive the expression for this coefficient from (E.2), one needs to permute the integrations with respect to $v$ and $u_{a}, u_{b}, u_{c}$. Because of the presence of the Cauchy principal value in $G_{k}(u, v)$, the Poincaré-Bertrand equality must be used. After a lenghty calculation, the details of which can be found in [62], one finds

$$
I=\frac{\mathrm{i} m k_{a} k_{b} k_{c}}{3(4 \pi e)^{3}} \int_{-\infty}^{\infty} j\left(u_{a}, u_{b}, u_{c}, v\right) \mathrm{d} v,
$$

with

$$
\begin{aligned}
j & =\frac{1}{f_{0}^{\prime 2}} G_{k_{a}}\left(u_{a}, v\right) G_{k_{b}}\left(u_{b}, v\right) G_{k_{c}}\left(u_{c}, v\right) \\
& +\frac{1}{f_{0}^{\prime 2}}\left[\varepsilon_{I}\left(k_{a}, v\right) \varepsilon_{I}\left(k_{b}, v\right) \delta\left(v-u_{a}\right) \delta\left(v-u_{b}\right) G_{k_{c}}\left(u_{c}, v\right)+\text { cyc. }\right]
\end{aligned}
$$

where cyc designates the sum of the three cyclic permutations of $(a, b, c)$. 


\section{References}

[1] V. I. Arnol'd, V. V. Kozlov, and A. I. Neishtadt. Mathematical Aspects of Classical and Celestial Mechanics. Encyclopedia of Mathematical Sciences: Dynamical Systems III. Springer-Verlag, 1988.

[2] J. Henrard. The adiabatic invariant in classical mechanics. In C. K. R. T. Jones, U. Kirchgraber, and H. O. Walther, editors, Dynamics Reported 2, pages 117-235. Springer-Verlag, 1993.

[3] P. J. Morrison and J. M. Greene. Noncanonical Hamiltonian density formulation of hydrodynamics and ideal magnetohydrodynamics. Phys. Rev. Lett., 45:790-793, 1980. Erratum: 48, 569 (1982).

[4] P. J. Morrison. Poisson brackets for fluids and plasmas. In M. Tabor and Y. Treve, editors, Mathematical Methods in Hydrodynamics and Integrability in Dynamical Systems, volume 88 of American Institute of Physics Conference Proceedings, pages 13-45. American Institute of Physics, 1982.

[5] P. J. Morrison. Hamiltonian description of the ideal fluid. Rev. Mod. Phys., 70:467-521, 1998.

[6] C. Carathéodory. Calculus of variations and partial differential equations of first order. HoldenDay, 1965.

[7] L. P. Eisenhart. Continuous groups of transformations. Dover, 2003.

[8] J. E. Marsden and A. Weinstein. Reduction of symplectic manifolds with symmetry. Rep. Math. Phys., 5:121-130, 1974.

[9] J. E. Marsden and T. S. Ratiu. Introduction to mechanics and symmetry. Springer-Verlag, 1999.

[10] R. G. Littlejohn. A guiding center Hamiltonian: a new approach. J. Math. Phys., 20:2445-2458, 1979.

[11] H. Samelson. Darboux's lemma once more. Proc. Am. Math. Soc., 123:1253-1255, 1995.

[12] C. S. Kueny and P. J. Morrison. Nonlinear instability and chaos in plasma wave-wave interactions. I. Introduction. Phys. Plasmas, 2:1926-1940, 1995.

[13] A. Weinstein. The local structure of Poisson manifolds. J. Diff. Geom., 22:523-557, 1983. Erratum: 22, 255 (1985).

[14] J. F. Conn. Normal forms for smooth Poisson structures. Ann. Mat., 121:565-593, 1985.

[15] V. I. Arnol'd and A. B. Givental. Symplectic Geometry. Encyclopedia of Mathematical Sciences: Dynamical Systems IV. Springer-Verlag, 1990.

[16] R. C. Davidson. Methods in Nonlinear Plasma Theory. Academic Press, Inc., 1972.

[17] V. E. Zakharov, V. S. L'vov, and G. Falkovich. Kolmogorov Spectra of Turbulence 1. Wave Turbulence. Springer-Verlag, 1992.

[18] G. D. Birkhoff. Relativity and Modern Physics. 2nd. edition. Harvard University Press, 1927.

[19] C. R. Rao and S. K. Mitra. Generalized inverse of matrices and its applications. John Wiley and Sons, 1971.

[20] N. Jacobson. Lie Algebras. John Wiley Interscience, 1962.

[21] R. Gilmore. Lie groups, Lie algebras, and some of their applications. Dover, 2002.

[22] E. Sudarshan and N. Mukunda. Classical Dynamics: a Modern Perspective. John Wiley, 1974.

[23] K. Ngan, S. Meacham, and P. J. Morrison. Elliptical vortices in shear: Hamiltonian moment formulation and melnikov analysis. Phys. Fluids, 4:896-913, 1996. 
[24] S. P. Meacham, P. J. Morrison, and G. R. Flierl. Hamiltonian moment reduction for describing vortices in shear. Phys. Fluids, 9:2310-2328, 1997.

[25] J. L. Thiffeault and P. J. Morrison. Classification and Casimir invariants of Lie-Poisson brackets. Physica D, 136:205-244, 2000.

[26] P. J. Olver. A nonlinear Hamiltonian structure for the Euler equations. J. Math. Anal. Appl., 89:223-250, 1982.

[27] J. E. Marsden and A. Weinstein. Coadjoint orbits, vortices and Clebsch variables for incompressible fluids. Physica D, 7:305-323, 1983.

[28] P. J. Morrison. The Maxwell-Vlasov equations as a continuous Hamiltonian system. Phys. Lett. A, 80:383-, 1980 .

[29] P. J. Morrison and R. D. Hazeltine. Hamiltonian formulation of reduced magnetohydrodynamics. Phys. Fluids, 27:886-897, 1984.

[30] J. E. Marsden and P. J. Morrison. Noncanonical Hamiltonian field theory and reduced MHD. Contemporary Mathematics, 28:133-150, 1984.

[31] O. Bühler. Waves and mean flows. Cambridge Univ. Press, 2009.

[32] T. G. Shepherd. Symmetries, conservation laws and Hamiltonian structure in geophysical fluid dynamics. Adv. Geophys., 32:287-338, 1990.

[33] N. J. Balmforth and P. J. Morrison. Hamiltonian description of shear flow. In J. Norbury and I. Roulstone, editors, Large-Scale Atmosphere-Ocean Dynamics II, pages 117-142. Cambridge, 2002.

[34] P. J. Morrison and D. Pfirsch. Free energy expressions for Vlasov-Maxwell equilibria. Phys. Rev. A., 40:3898-, 1989.

[35] V. Zakharov and L.I.Piterbarg. Canonical variables for Rossby waves and plasma drift waves. Phys. Lett. A, 126:497-500, 1988.

[36] D. D. Holm, J. E. Marsden, T. Ratiu, and A. Weinstein. Nonlinear stability of fluid and plasma equilibria. Phys. Repts., 123:1-116, 1985.

[37] T. B. Benjamin. On the Boussinesq model for two-dimensional wave motions in heterogeneous fluids. J. Fluid Mech., 165:445-474, 1986.

[38] P.J. Morrison, I. L. Caldas, and H. Tasso. Hamiltonian formulation of two-dimensional gyroviscous MHD. Zeit. Naturforsch., 39a:1023-, 1985.

[39] R. D. Hazeltine, C. T. Hsu, and P. J. Morrison. Hamiltonian four-field model for nonlinear tokamak dynamics. Phys. Fluids, 30:3204-3211, 1987.

[40] T. J. Schep, F. Pegoraro, and B. N. Kuvshinov. Generalized two-fluid theory of nonlinear magnetic structures. Phys. Plasmas, 1:2843-2851, 1994.

[41] B. N. Kuvshinov, F. Pegoraro, and T. J. Schep. Hamiltonian formulation of low-frequency, nonlinear plasma dynamics. Phys. Lett. A, 191:296-300, 1994.

[42] D. Grasso, F. Califano, F. Pegoraro, and F. Porcelli. Phase mixing and saturation in Hamiltonian reconnection. Phys. Rev. Lett., 86:5051-5054, 1994.

[43] J. A. Krommes and R. A. Kolesnikov. Hamiltonian description of convective-cell generation. Phys. Plasmas, 11:L29-L32, 2004.

[44] F. L. Waelbroeck, P. J. Morrison, and W. Horton. Hamiltonian formulation and coherent structures in electrostatic turbulence. Plasma Phys. and Control. Fusion, 46:1331-1350, 2004.

[45] E. Tassi, P. J. Morrison, F. L. Waelbroeck, and D. Grasso. Hamiltonian formulation and analysis of a collisionless fluid reconnection model. Plasma Phys. and Control. Fusion, 50:085014, 2008. 
[46] I. Keramidas Charidakos, F. Waelbroeck, and P. J. Morrison. Hamiltonian five-field gyrofluid model. Phys. Plasmas, 2015.

[47] F. L. Waelbroeck, R. D. Hazeltine, and P. J. Morrison. Hamiltonian electromagnetic gyrofluid model. Phys. Plasmas, 16:032109, 2009.

[48] F. L. Waelbroeck and E. Tassi. A compressible Hamiltonian electromagnetic gyrofluid model. Comm. Nonlin. Sci. Num. Sim., 17:2171-2178, 2012.

[49] E. Tassi, P. J. Morrison , D. Grasso, and F. Pegoraro. Hamiltonian four-field model for magnetic reconnection: nonlinear dynamics and extension to three dimensions with externally applied fields. Nuc. Fusion, 50:034007, 2010.

[50] H. M. Abdelhamid, Y. Kawazura, and Z. Yoshida. Hamiltonian Formalism of Extended Magnetohydrodynamics. J. Phys. A, 48, 2015.

[51] M. Lingam, P. J. Morrison, and G. Miloshevich. Remarkable connections between extended magnetohydrodynamics models. Phys. Plasmas, 22:072111, 2015.

[52] Z. Yoshida and E. Hameiri. Canonical Hamiltonian mechanics of Hall magnetohydrodynamics and its limit to ideal magnetohydrodynamics. J. Phys. A, 46:G5502, 2013.

[53] P. J. Morrison. The Maxwell-Vlasov equations as a continuous Hamiltonian system. Phys. Lett. A, 80:383-386, 1980.

[54] J. E. Marsden and A. Weinstein. The Hamiltonian structure of the Maxwell-Vlasov equations. Physica D, 4:394-406, 1982.

[55] P. J. Morrison. A general theory for gauge-free lifting. Phys. Plasmas, 20:012104, 2013.

[56] S. Nguyen and L. A. Turski. Canonical description of incompressible fuid: Dirac brackets approach. Physica A, 272:48-55, 1999.

[57] C. Chandre, P. J. Morrison, and E. Tassi. On the Hamiltonian formulation of incompressible ideal fluids and magnetohydrodynamics via Dirac's theory of constraints. Phys. Lett. A, 46:737$743,2012$.

[58] C. Chandre, L. de Guillebon, A. Back, E. Tassi, and P. J. Morrison. On the use of projectors for Hamiltonian systems and their relationship with Dirac brackets. J. Phys. A, 376:125203, 2013.

[59] C. Chandre, P. J. Morrison, and E. Tassi. Hamiltonian formulation of the modified Hasegawa Mima equation. Phys. Lett. A, 378:956-959, 2014.

[60] C. Chandre. Casimir invariants and the Jacobi identity in Dirac's theory of constrained Hamiltonian systems. J. Phys. A, 46:375201, 2013.

[61] P. J. Morrison. Hamiltonian description of Vlasov dynamics: action-angle variables for the continuous spectrum. Trans. Theory Stat. Phys., 29:397-414, 2000.

[62] T. W. Yudichak. Hamiltonian methods in weakly nonlinear Vlasov-Poisson Dynamics. Ph.D. Thesis, University of Texas at Austin, 2001.

[63] J. Vanneste. Rossby wave interactions in a shear flow with critical levels. J. Fluid Mech., 323:317-338, 1996.

[64] J. Vanneste. A nonlinear critical layer generated by the interaction of free Rossby waves. $J$. Fluid Mech., 371:319-344, 1998.

[65] J. Vanneste, P. J. Morrison, and T. Warn. Strong echo effect and nonlinear transient growth in shear flows. Phys. Fluids, 10:1398-1404, 1998.

[66] J. Bedrossian and N. Masmoudi. Inviscid damping and the asymptotic stability of planar shear flows in the 2D Euler equations. Publications mathématiques de l'IHES (published online), arXiv:1306.5028, 2013. 
[67] J. Weiland and H. Wilhelmsson. Coherent Nonlinear Interaction of Waves in Plasmas. Pergamon Press, New York, 1977.

[68] G. I. Hagstrom and P. J. Morrison. On Krein-like theorems for noncanonical Hamiltonian systems with continuous spectra: application to Vlasov-Poisson. Trans. Theory Stat. Phys., 39:466-501, 2010.

[69] T. F. Visconti, I. L. Caldas, and P. J. Morrison. A method for Hamiltonian truncation: a four-wave example. arXiv:1509.09247v1, 2015.

[70] T. F. Visconti, I. L. Caldas, and P. J. Morrison. work in progress, 2015.

[71] P. J. Morrison and D. Pfirsch. Dielectric energy versus plasma energy, and Hamiltonian actionangle variables for the Vlasov equation. Phys. Fluids B, 4:3038-3057, 1992.

\section{References}

